\title{
Evaluation of an Analytical Model for the Maximum Intensity of Tropical Cyclones
}

\author{
GEORGE H. BRYAN AND RiCHARD ROTUNNO \\ National Center for Atmospheric Research, * Boulder, Colorado
}

(Manuscript received 17 December 2008, in final form 24 April 2009)

\begin{abstract}
Several studies have shown that the intensity of numerically simulated tropical cyclones can exceed (by $50 \%$ ) a theoretical upper limit. To investigate the cause, this study evaluates the underlying components of Emanuel's commonly cited analytic theory for potential intensity (herein referred to as E-PI). A review of the derivation of E-PI highlights three primary components: a dynamical component (gradient-wind and hydrostatic balance); a thermodynamical component (reversible or pseudoadiabatic thermodynamics, although the pseudoadiabatic assumption yields greater intensity); and a planetary boundary layer (PBL) closure (which relates the horizontal gradients of entropy and angular momentum at the top of the PBL to fluxes and stresses at the ocean surface). These three components are evaluated using output from an axisymmetric numerical model. The present analysis finds the thermodynamical component and the PBL closure to be sufficiently accurate for several different simulations. In contrast, the dynamical component is clearly violated. Although the balanced portion of the flow ( $v_{g}$, to which E-PI applies) appears to also exceed E-PI, it is shown that this difference is attributable to the method used to calculate $v_{g}$ from the model output. Evidence is shown that $v_{g}$ for a truly balanced cyclone does not exceed E-PI. To clearly quantify the impact of unbalanced flow, a more complete analytic model is presented. The model is not expressed in terms of external conditions and thus cannot be used to predict maximum intensity for a given environment; however, it does allow for evaluation of the relative contributions to maximum intensity from balanced and unbalanced (i.e., inertial) terms in the governing equations. Using numerical model output, this more complete model is shown to accurately model maximum intensity. Analysis against observations further confirms that the effects of unbalanced flow on maximum intensity are not always negligible. The contribution to intensity from unbalanced flow can become negligible in axisymmetric models as radial turbulence (i.e., viscosity) increases, and this explains why some previous studies concluded that E-PI was an accurate upper bound for their simulations. Conclusions of this study are also compared and contrasted to those from previous studies.
\end{abstract}

\section{Introduction}

Several recent studies have found that numerically simulated tropical cyclone intensity can exceed theoretical estimates for maximum possible intensity. For example, Persing and Montgomery [2003 (hereafter PM03), 2005], Hausman et al. (2006), Cram et al. (2007), Yang et al. (2007), and Bryan and Rotunno [2009a,b (hereafter BR09b)] all found that simulated maximum intensity can be larger, sometimes by $50 \%$, than the maximum possible

\footnotetext{
* The National Center for Atmospheric Research is sponsored by the National Science Foundation.
}

Corresponding author address: George H. Bryan, National Center for Atmospheric Research, 3450 Mitchell Lane, Boulder, CO 80301.

E-mail: gbryan@ucar.edu value predicted by the analytical model of Emanuel (1986, hereafter E86). The purpose of this article is to investigate the source of this discrepancy.

To this end, we evaluate the underlying assumptions of Emanuel's potential intensity theory (E-PI; E86) using output from numerical model simulations in which steady tropical cyclone intensity greatly exceeds E-PI. The same methodology was undertaken by PM03. They ultimately concluded that the underlying thermodynamic assumption in E-PI (moist slantwise neutrality) was not met in their modeled hurricane. To explain this finding, they implicated a mechanism that was not considered in E-PI: surface entropy fluxes in the hurricane eye and the subsequent exchange of entropy between the eye and eyewall. Further analysis by Bryan and Rotunno (2009a) found that the PM03 mechanism was quantitatively small in numerical model simulations and thus cannot explain a lack of moist slantwise neutrality in PM03's analyses. 
Another analysis of E-PI was provided by Smith et al. (2008, hereafter SMV08). They concluded that the lack of explicit consideration of the radial momentum budget in the boundary layer is a major shortcoming of E-PI and that E-PI makes a "tacit assumption" of gradient-wind balance in the boundary layer, which causes a systematic underprediction of intensity. They recommended the use of a more complete boundary layer model in E-PI.

In light of the results presented here, we further consider these analyses of E-PI in a later discussion section. For now, we note that the primary conclusions of these two studies are different. PM03 implicate primarily the underlying thermodynamic assumption (moist slantwise neutrality), whereas SMV08 implicate primarily the dynamic assumption (gradient-wind balance) and its coupling with the boundary layer. It is conceivable that all of these components of E-PI are deficient and that all contribute to the discrepancy. A comprehensive analysis of these processes, using a common dataset, seems warranted.

Additionally, there are reasonable concerns about the accuracy of numerical models used in previous studies. One numerical model, developed by Rotunno and Emanuel (1987, hereafter RE87) and used to evaluate E-PI by RE87, PM03, and others, has since been shown to have a positive intensity bias (of $\sim 10 \%$ ) that is attributable to several approximations in that code (BR09b). For our analysis, we use a numerical model that makes fewer approximations and uses more accurate numerical techniques. Another advantage of this model is that the underlying thermodynamics are formulated consistently with the thermodynamics used for E-PI, so a straightforward comparison can be made.

To put our analysis into context, we review in section 2 the primary underlying assumptions of E-PI. We then evaluate these assumptions using output from our numerical model in sections 3 and 4 . We identify only one assumption that is clearly not applicable to our model output, and thus we analyze this assumption in more detail in section 5 . We also advance a more complete analytic model and evaluate it using the numerical model in section 6. A discussion of these results in the context of past studies in provided in section 7 , and a brief synthesis of our conclusions is given in section 8 .

\section{Review of E-PI}

In this section, we briefly review the fundamental components of E-PI. We broadly follow the derivation in (E86), although some details are different because of insights gained in the intervening years [e.g., dissipative heating was added by Bister and Emanuel (1998, hereafter BE98)]. At times, we compare against the deriva- tion in Emanuel (1988, hereafter E88) which makes fewer approximations but which is rarely used as a model of maximum intensity, perhaps because of its greater complexity.

Conceptually, E86 assumes a steady, axisymmetric tropical cyclone that can be separated into two parts: 1) a free atmosphere, for which viscous terms are negligible, and 2) a boundary layer, in which viscous terms and surface interaction are important. We address these two parts separately in the next two subsections.

\section{a. Free atmosphere}

In the free atmosphere, the flow is assumed to be in hydrostatic balance and gradient-wind balance, which is expressed mathematically as

$$
\begin{aligned}
& \frac{\alpha_{d}}{(1+Q)} \frac{\partial p}{\partial z}=-g \text { and } \\
& \frac{\alpha_{d}}{(1+Q)} \frac{\partial p}{\partial r}=\frac{M_{g}^{2}}{r^{3}}-\frac{1}{4} f^{2} r,
\end{aligned}
$$

where $r$ is radius, $z$ is height, $p$ is pressure, $\alpha_{d}$ is the specific volume of dry air, $Q \equiv q_{v}+q_{l}$ is the mixing ratio of total water, $q_{v}$ and $q_{l}$ are mixing ratios of water vapor and condensate, respectively, $g$ is gravitational acceleration, $M_{g} \equiv r v_{g}+f r^{2} / 2$ is angular momentum, $v_{g}$ is gradient wind speed, and $f$ is the Coriolis parameter. Here, we use the subscript $g$ to clarify explicitly that this analytic model imposes gradient-wind balance. Using (1) and (2), an equation for thermal-wind balance can be derived:

$$
\frac{1}{r^{3}}\left(\frac{\partial M_{g}^{2}}{\partial p}\right)_{r}=-\left\{\frac{\partial}{\partial r}\left[\alpha_{d}(1+Q)^{-1}\right]\right\}_{p},
$$

where pressure is now used as the vertical coordinate.

At this point, E86 makes an assumption about the thermodynamics that apply in the free atmosphere. For simplicity, the ice phase is not considered. Also for simplicity, one could make one of two extreme assumptions about the condensate: 1) reversible thermodynamics, in which all condensate moves with the air, or 2) pseudoadiabatic thermodynamics, in which all condensate is immediately removed from the air by fallout upon formation. Using axisymmetric numerical model simulations, BR09b found that the pseudoadiabatic assumption yields the greatest intensity. Because the ultimate goal of potential intensity (PI) theory is to derive the maximum realizable intensity, we choose the pseudoadiabatic assumption for the following derivation.

Before proceeding, we note that E88 assumed reversible thermodynamics and derived analogous relations 
for the free atmosphere (see section 2a in E88) without any approximations. In contrast, E86 neglected condensate loading and used an approximate first law of thermodynamics. Although E86 stated that reversible thermodynamics was assumed, it is clear that the E86 derivation is actually something different. Our derivation below clarifies this.

We use the first law of thermodynamics for pseudoadiabatic conditions (Bryan 2008) to derive an appropriate Maxwell relation. Details are provided in appendix A. We find $\left(\partial \alpha_{d} / \partial s\right)_{p}=(\partial T / \partial p)_{s}$, where $T$ is temperature and $s$ is total moist entropy. ${ }^{1}$ Additionally, under the pseudoadiabatic assumption we may assume that $\alpha_{d}$ is a function of only two other state variables: $p$ and $s$. Consequently, (3) can be rewritten, utilizing (A5), as

$$
\begin{aligned}
\frac{1}{r^{3}}\left(\frac{\partial M_{g}^{2}}{\partial p}\right)_{r}= & -\frac{1}{\left(1+q_{v}\right)}\left(\frac{\partial T}{\partial p}\right)_{s}\left(\frac{\partial s}{\partial r}\right)_{p} \\
& +\frac{\alpha_{d}}{\left(1+q_{v}\right)^{2}}\left(\frac{\partial q_{v}}{\partial r}\right)_{p}
\end{aligned}
$$

where we have assumed $q_{l}=0$ (consistent with the pseudoadiabatic assumption).

We next assume that the final term in (4) may be reasonably neglected. This approximation seems to be required for further development of a pseudoadiabatic PI model because $q_{v}$ is not a conserved variable, and hence this term is not easily integrable (which is the next step in the derivation). In contrast, an analogous term in the reversible formulation (see E88) is easily integrable because $Q$ is conserved in that system. E88's reversible derivation ultimately yields a term (proportional to $d Q / d M_{g}$ ) that has no counterpart in E86's derivation.

Viewed another way, we may assume that $1 /\left(1+q_{v}\right) \approx$ 1 in (1) and (2) (with $q_{l}=0$, owing to the pseudoadiabatic assumption). In other words, we neglect the mass of water vapor in the momentum equations. We find from numerical simulations (not shown) that this approximation has negligible impact on the structure and intensity of heavily precipitating tropical cyclones; the maximum difference in $v$ was $6 \%$ in our tests. Thus, if we neglect $q_{v}$ from the momentum equations at the beginning of the derivation, then we can repeat the derivation above and arrive without any further assumptions at

$$
\frac{1}{r^{3}}\left(\frac{\partial M_{g}^{2}}{\partial p}\right)_{r}=-\left(\frac{\partial T}{\partial p}\right)_{s}\left(\frac{\partial s}{\partial r}\right)_{p}
$$

\footnotetext{
${ }^{1}$ The formulation for $s$ used herein applies to both saturated and unsaturated conditions; hence, there is no need for special notation for the saturated state, as was done in E86.
}

which is identical to E86's (8). We consider the neglect of water vapor mass from the momentum equations to be a reasonable, if not rigorously justified, approximation.

The derivation next assumes that $s$ does not vary along $M_{g}$ surfaces (thus, $s$ is a function of $M_{g}$ only). This conclusion is valid if viscous effects are negligible in the free atmosphere because both $s$ and $M_{g}$ would be conserved along streamlines. As discussed in E86, these conditions also imply a state of moist slantwise neutrality. The mathematical expression (5) may thus be expressed without further approximation as

$$
\left(\frac{\partial r}{\partial p}\right)_{M_{g}}=\frac{r^{3}}{2 M_{g}} \frac{d s}{d M_{g}}\left(\frac{\partial T}{\partial p}\right)_{s}
$$

which is an expression for the slope of streamlines in the free atmosphere. We note that (6) can be obtained directly from E88's (7) (the unapproximated reversible formulation) by assuming $Q=0$ everywhere; this further supports our interpretation that the approximations made by E86 are not consistent with reversible thermodynamics.

Considering now a specific trajectory in the flow, (6) may be integrated upward from an arbitrary point $(r, p)$ to some point at large radius and small pressure $\left(r_{\text {out }}\right.$, $\left.p_{\text {out }}\right)$ at which the trajectory becomes essentially horizontal. Considering the trajectory that passes through the maximum gradient wind $\left(v_{g, \max }\right)$, assuming also that the contribution to $M_{g}$ from the earth's rotation is negligible at this location, and further assuming that $r_{\text {out }} \gg$ $r_{\max }$, then this expression can be derived:

$$
v_{g, \text { max }}^{2}=-M_{g} \frac{d s}{d M_{g}}\left(T_{B}-T_{\text {out }}\right) \quad \text { at } \quad(r, z)=\left(r_{\max }, z_{\max }\right),
$$

where $\left(r_{\max }, z_{\max }\right)$ is the location of $v_{g, \max }, T_{B}$ is temperature at this location, and $T_{\text {out }}$ is temperature at $r_{\text {out }}$ along this assumed trajectory. [This is essentially (12) of E86, with the only change being the use of $M_{g} \approx$ $r_{\max } v_{g, \text { max }}$ to write the expression in terms of $v_{g \text {,max }}$.] This completes the derivation for the free atmosphere.

Broadly speaking, only two primary components underlie (7): a dynamical component and a thermodynamical component. The assumption of gradient-wind and hydrostatic balance (i.e., thermal-wind balance) constitutes the dynamical component. The assumption of pseudoadiabatic thermodynamics constitutes the thermodynamical component. We evaluate the applicability of both assumptions using numerical model output later in this article. We made several other approximations in our derivation of (7), including the neglect of water vapor 
mass from the momentum equations (which seems to be reasonable, according to numerical simulations) and the neglect of viscous effects (which is an important factor in simulated intensity, as discussed by BR09b; we return to this point in a later discussion section).

Finally, the impact of some approximations made by E86 has never been clear. Because we obtain the same relation as E86, we conclude that E86's relation essentially applies to pseudoadiabatic thermodynamic conditions. We reiterate that the accurate (unapproximated) solution for reversible thermodynamics was derived by E88.

\section{b. Boundary layer}

Next, E86 turns to a simple planetary boundary layer (PBL) model to couple the free atmosphere to conditions at the ocean surface. The derivation considers the variables $s, M$, and radial velocity $(u)$ to be well mixed (i.e., constant) in the PBL. It is further assumed that steady values of $s$ and $M$ are maintained by a balance between radial advection and vertical turbulent transport. BE98 extended the E86 PBL model to account for dissipative heating (the increase in internal energy that occurs when kinetic energy is dissipated). We make the same assumptions as BE98, the most notable being that dissipative heating is only important in the boundary layer and that all of the heating goes into warming the atmosphere (not the ocean). Using equations that account for these processes (see BE98), we integrate from the surface to the top of the boundary layer. Assuming that turbulent fluxes go to zero at $z=h$, then we obtain

$$
\left.\frac{\partial s}{\partial M}\right|_{z=h}=-\left.\frac{\tau_{s}}{\tau_{M}}\right|_{z=0}-\frac{\int_{0}^{h} \epsilon / T d z}{\left.\tau_{M}\right|_{z=0}}
$$

where $\tau_{s}$ and $\tau_{M}$ are, respectively, the fluxes of $s$ and $M$ at the sea surface, and $\varepsilon$ is the rate of dissipative heating. Using bulk aerodynamic formulas for surface fluxes and stresses, and assuming that $(\varepsilon / T)$ decreases linearly from its value at the surface to zero at $z=h$, this relation can be expressed as

$$
\left.\frac{\partial s}{\partial M}\right|_{z=h}=-\frac{C_{E}}{C_{D}} \frac{\left(s_{\text {surf }}-s_{0}\right)}{r v_{0}}-\frac{1}{T_{s}} \frac{|V|^{2}}{r v_{0}},
$$

where $C_{E}$ and $C_{D}$ are the surface exchange coefficients for entropy and momentum, respectively, $s_{\text {surf }}$ is entropy at saturation at sea surface temperature $\left(T_{s}\right), s_{0}$ is entropy at the top of the surface layer, $|V|$ is the magnitude of (total) velocity at the top of the surface layer, and $r v_{0}$ is $M$ at the top of the surface layer (neglecting the con- tribution from the earth's rotation because this closure will be applied at the eyewall).

The result from the free atmosphere, (7), can then be coupled to the PBL closure, (9), by assuming that the radial gradient of $M$ at the top of the boundary layer is approximately equal to the radial gradient in $M_{g}$ (i.e., $\left.\partial M / \partial M_{g} \approx 1\right)$ and that $v_{g \text {,max }}$ is located at the top of the boundary layer (i.e., at $z=h$, where viscous terms become negligible). To make the final equation relatively simple, we also need to assume $\left.v_{g}\right|_{z=h} \approx|V| \approx v_{0}$, which is consistent with the assumption of a well-mixed boundary layer. Assuming also that $T_{s} \approx T_{B}$, these steps then yield the relation

$$
v_{g, \text { max }}^{2}=\alpha \frac{C_{E}}{C_{D}}\left(T_{B}-T_{\text {out }}\right)\left(s_{\text {surf }}-s_{0}\right) \text { at }\left(r_{\max }, z_{\max }\right),
$$

where $\alpha \equiv T_{s} / T_{0}$ is a term associated with dissipative heating. ${ }^{2}$ This completes the derivation of E-PI as it is evaluated in this article. We note that (10) is equivalent to equations used by PM03 and others, although here we clarify that it applies to the maximum gradient wind speed.

Our review of the PBL component has identified several assumptions and approximations. The fundamental closure, however, can be expressed succinctly by (8). We evaluate this closure using numerical model output later in this article. As for several seemingly minor approximations, such as the assumed linear vertical structure of $\varepsilon / T$ and the approximations $\left.v_{g}\right|_{z=h} \approx$ $|V| \approx v_{0}$, in the interest of brevity we defer evaluation of these approximations to a future study.

\section{c. Summary}

Hereafter, we evaluate this analytic model of maximum intensity using output from an axisymmetric numerical model. Quantitatively, we calculate $(\mathrm{E}-\mathrm{PI})^{2}$ using the right side of (10). This technique was also used by PM03, and it requires evaluation of terms on the right side of (10) at the location of maximum azimuthal velocity in a steady tropical cyclone. Note that this method does not produce an estimate of maximum possible intensity; rather, it evaluates the ability of (10) to model the simulated tropical cyclones.

From the derivation above, we identify three primary components of E-PI that require evaluation:

- gradient-wind and hydrostatic balance (i.e., thermalwind balance) in the free atmosphere;

\footnotetext{
${ }^{2}$ For the sake of comparison to previous studies, we note that if dissipative heating is not considered (as in E86) then the final term on the right sides of (8) and (9) is zero, and it follows that $\alpha=1$.
} 
- moist slantwise neutrality (assuming pseudoadiabatic thermodynamics) in the free atmosphere; and

- a PBL closure, as represented by (8).

We reiterate that several other approximations have been used to derive E-PI. We consider these to be relatively good approximations, and the following analysis supports this conjecture.

\section{Description of numerical simulations}

For all simulations, we use the axisymmetric numerical model of BR09b. We use only the "conservative equation set" (see BR09b) that permits conservation of total mass and internal energy in saturated flow. Dissipative heating is included unless stated otherwise, and the surface exchange coefficients are formulated the same way as in RE87 such that $C_{E} / C_{D}=1$. The domain size is $1500 \mathrm{~km} \times 25 \mathrm{~km}$, the vertical grid spacing is $250 \mathrm{~m}$, and the radial grid spacing is $1 \mathrm{~km}$ for $r<64 \mathrm{~km}$ (with stretched radial grid spacing for $r>64 \mathrm{~km}$, as in BR09b, to decrease computational expense). All simulations use the simple relaxation term that mimics radiative cooling, and this term is capped at $2 \mathrm{~K} d a y^{-1}$.

\section{a. Control simulation}

BR09b investigated the settings in this model that yield greatest hurricane intensity. They found that pseudoadiabatic thermodynamics (i.e., instantaneous removal of condensate from the atmosphere upon formation) and inviscid flow (in the free atmosphere) yielded greatest intensity. These two conditions are also assumed by E-PI, as reviewed in the previous section. Thus, for the sake of fair comparison, our control simulation uses a small horizontal turbulence length scale $\left(l_{h}=94 \mathrm{~m}\right)$, and we immediately remove most condensate (in excess of $\left.0.1 \mathrm{~g} \mathrm{~kg}^{-1}\right){ }^{3}$ The vertical turbulence length scale $\left(l_{v}\right)$ is set to $100 \mathrm{~m}$.

The sea surface temperature $\left(T_{s}\right)$ is fixed at $28^{\circ} \mathrm{C}$. In the spirit of RE87's study, we set the environment to be exactly neutral to moist convection. The initial sounding for the control simulation (Fig. 1) is saturated and was constructed assuming constant pseudoadiabatic equivalent potential temperature using the model's equations without approximation. The surface temperature for this sounding is $3^{\circ} \mathrm{C}$ colder than $T_{s}$, which is consistent

\footnotetext{
${ }^{3}$ We do not set $l_{h}$ to zero because some weak horizontal diffusion is needed to produce realistic flow in the eye of these simulations, and we permit a small amount of liquid in the domain to ensure that weakly subsiding flow remains saturated. Simulations with $l_{h}=0$ and immediate removal off all condensate yield essentially the same intensity but produce some undesirable flow structures.
}

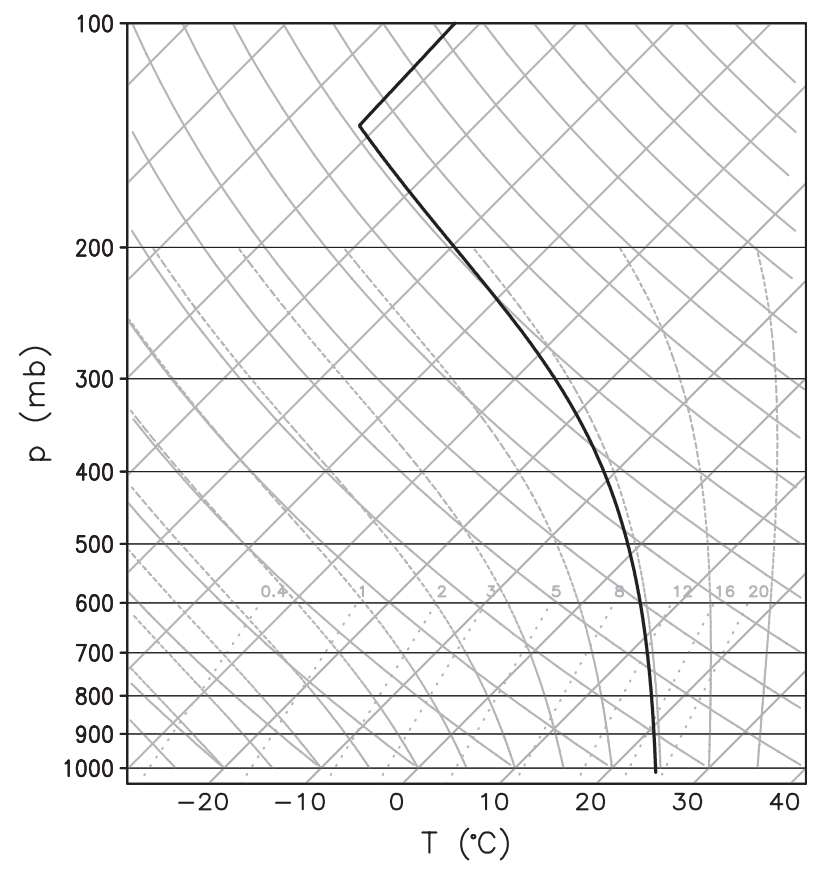

FIG. 1. The initial environmental sounding for the control simulation. The environment is saturated at all levels.

with observations in the core of intense tropical cyclones (e.g., Cione et al. 2000; LeeJoice 2000). The initial vortex is identical to that in RE87.

In this simulation, the maximum azimuthal velocity increases rapidly, reaching almost $120 \mathrm{~m} \mathrm{~s}^{-1}$ in 2 days (Fig. 2). A slight weakening occurs over the next several days, and then an approximately steady intensity is produced for $t>4$ days. We quantify the intensity of tropical cyclones in this article by $v_{\max }$, which is the average of the maximum value of $v$ from every time step during $t=4-8$ days. For the control simulation, $v_{\max }=$ $108 \mathrm{~m} \mathrm{~s}^{-1}$. For other analyses herein, we utilize the average state computed from hourly output during $t=4-8$ days. Throughout this article, we evaluate only maximum hurricane intensity in steady state because this is what E-PI addresses. The dynamics of hurricane intensification were addressed recently by Smith et al. (2009; see also references therein).

To determine E-PI, we use the right side of (10), where all terms are evaluated at the location of maximum winds $\left(r_{m}, z_{m}\right)$, except $s_{0}$ is evaluated at the top of the surface layer (i.e., at the lowest model level). For $T_{\text {out }}$, we compute a trajectory (from the time-averaged flow fields) that starts at $\left(r_{m}, z_{m}\right)$; we follow this trajectory to a point far downstream and average $T$ along this trajectory between $150 \mathrm{~km}<r<200 \mathrm{~km}$ to obtain $T_{\text {out }}$. For the control simulation, we find E-PI $=72 \mathrm{~m} \mathrm{~s}^{-1}$, which is $\sim 35 \%$ weaker than $v_{\max }$, consistent with previous studies of E-PI from model simulations (e.g., 


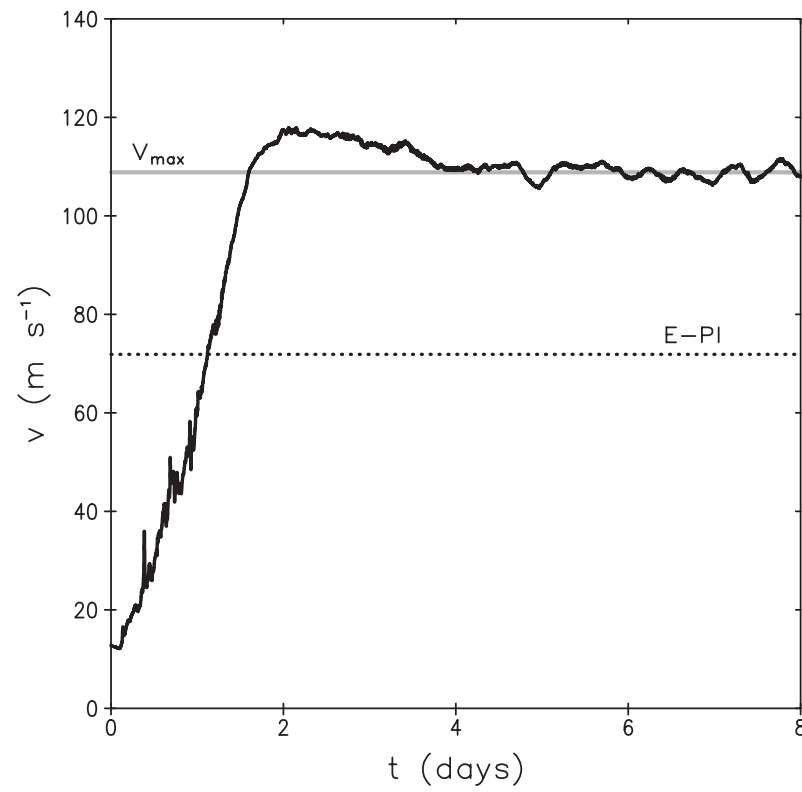

FIG. 2. Time series of maximum azimuthal velocity $\left(\mathrm{m} \mathrm{s}^{-1}\right)$ from the control simulation; $v_{\max }$ is the average value between $t=4-8$ days E-PI is indicated as a horizontal dotted line for reference.

PM03; Persing and Montgomery 2005; Cram et al. 2007; Yang et al. 2007; Bryan and Rotunno 2009a).

As discussed in the previous section, E-PI is an estimate of the maximum gradient wind speed $\left(v_{g \text { max }}\right)$. Following standard convention, we compute $v_{g}$ from the model output by writing an equation for gradient wind balance-similar to (2), but using this model's radial momentum equation-and then solving for $v_{g}$ :

$$
v_{g}=-\frac{f r}{2}+\left(\frac{f^{2} r^{2}}{4}+r c_{p} \theta_{v} \frac{\partial \pi}{\partial r}\right)^{1 / 2}
$$

where $c_{p}$ is the specific heat of dry air at constant pressure, $\theta_{v}$ is virtual potential temperature, and $\pi$ is nondimensional pressure (see BR09b for details). We show in Fig. 3 the radial distributions of $v$ and $v_{g}$ at the top of the boundary layer $\left(z=z_{m}=1.1 \mathrm{~km}\right)$. The shaded region in this figure denotes the eyewall, defined as $w>$ $0.5 \mathrm{~m} \mathrm{~s}^{-1}$ at this level. The maximum value of $v_{g}$ is $91 \mathrm{~m} \mathrm{~s}^{-1}$, and it is located at the outside edge of the eyewall (consistent with where E86 assumes $v_{g \text {,max }}$ is located); this is $\sim 25 \%$ stronger than E-PI, which reinforces the conclusion that E-PI is systematically too low.

Because E-PI (as determined above) has been calculated at the location of $v_{\max }$, we have also calculated it at the location of $v_{g \text {,max }}$, to check whether there could be a difference that is attributable to methodology. At the location of $v_{g, \text { max }}$, we find E-PI $=67 \mathrm{~m} \mathrm{~s}^{-1}\left(5 \mathrm{~m} \mathrm{~s}^{-1}\right.$ lower than E-PI computed at the location of $v_{\max }$ ), which

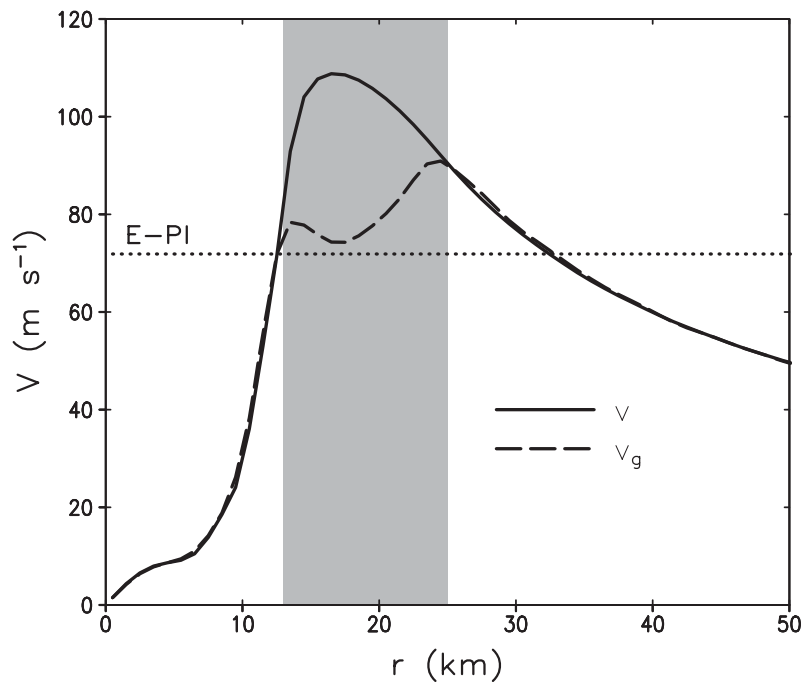

FIG. 3. Model output at the top of the boundary layer $(z=1.1 \mathrm{~km})$ : $v$ (solid), $v_{g}$ (dashed), and the location of the eyewall (shaded region). E-PI, which is calculated at the location of maximum $v$, is indicated by a horizontal dotted line for reference.

further reinforces the conclusion that E-PI is systematically lower than the intensity of the hurricane in this simulation. Hereafter, we compute E-PI only at the location of $v_{\text {max }}$

\section{b. Additional simulations}

As a check on the generality of our results, we conduct some analyses using the simulations from BR09b. These simulations use the same domain size, grid spacing, and initial vortex, but they use a different environment (see Fig. 1 in BR09b; $T_{s}=26.1^{\circ} \mathrm{C}$ ), a value of $l_{v}=200 \mathrm{~m}$, and a finite fall velocity for condensate $\left(V_{t}=7 \mathrm{~m} \mathrm{~s}^{-1}\right)$ (i.e., they do not impose pseudoadiabatic thermodynamics). Further details are provided in BR09b. To obtain a sufficiently broad range of results, we present analyses from several simulations that have used different values for $l_{h}$, which is the model parameter that has the greatest effect on hurricane structure and intensity. Because these simulations take more time to reach a steady state, we conduct these analyses using output from $t=8-12$ days.

\section{Evaluation of E-PI}

To determine why E-PI is too low in our numerical simulations, we evaluate the three primary components of E-PI in the next several subsections. We note that the hypothesis advanced by PM03 has been addressed in another study (Bryan and Rotunno 2009a) and thus we do not discuss it further in this article.

In the following, we do not address the realism of the numerical simulations or whether the E-PI components are appropriate for natural tropical cyclones. Indeed, as 

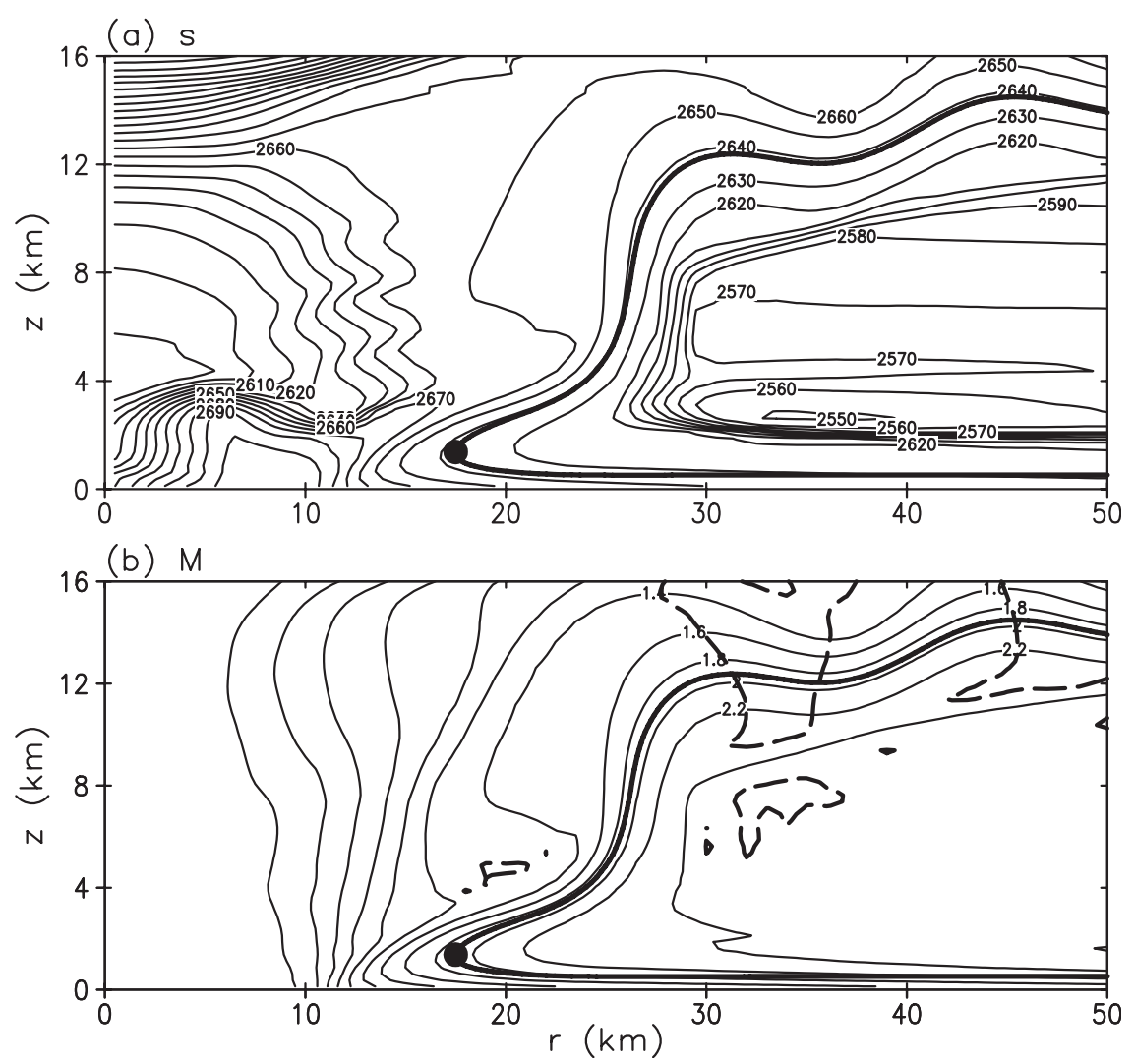

FIG. 4. Analysis of the trajectory that passes through $v_{\max }$ plotted over (a) $s$ (with contour interval of $10 \mathrm{~J} \mathrm{~kg}^{-1} \mathrm{~K}^{-1}$ ) and (b) $M$ (with contour interval of $0.2 \times 10^{6} \mathrm{~m}^{2} \mathrm{~s}^{-1}$ ). The trajectory is illustrated by the thick line, and the dot denotes the location of $v_{\max }$. The dashed contours in (b) enclose regions of inertial instability.

discussed in BR09b, tropical cyclones simulated with the control setup have some obviously unnatural features, such as intensity that exceeds the maximum observed intensity for a given sea surface temperature and inertial instability near the eyewall and upper-level outflow. (See BR09b for more details about model settings that are needed to obtain realistic tropical cyclone structures.) Rather, what we seek to evaluate is whether the inherent assumptions in E-PI can be realized in a numerical simulation and how any differences affect the maximum possible intensity in numerical simulations.

\section{a. Moist slantwise neutrality}

As demonstrated in section 2a, the E-PI relation can be derived by assuming pseudoadiabatic thermodynamical processes in the free atmosphere, which would be most useful for a theoretical upper bound on intensity (as demonstrated with numerical simulations by BR09b). Furthermore, E86 assumes that $s^{*}$ is a function of $M$ alone in the free atmosphere. As discussed further in E88 (p. 1144), this assumption may be viewed equivalently in two ways: 1) that the free atmosphere is neutral to slantwise convection or 2) that $s^{*}$ and $M$ are conserved variables that are constant along trajectories.

In Fig. 4, we show the trajectory that passes through the location of maximum winds in the control simulation. We also show the distribution of $s$ (Fig. 4a) as formulated for pseudoadiabatic processes (Bryan 2008) and the distribution of $M$ (Fig. 4b). To a good approximation, both $s$ and $M$ are conserved along streamlines in the free atmosphere, as determined by the near-equivalence of the trajectories and contours of $s$ or $M$. We draw the same conclusion from other trajectories in the eyewall (not shown). Further analysis of the budgets of $s$ and $M$ along these trajectories (not shown) reveals that $s$ and $M$ are not perfectly conserved, owing primarily to weak diffusion from the turbulence parameterization (particularly from vertical diffusion). Nevertheless, to a good approximation, $s$ and $M$ can be considered nearly constant along trajectories in the eyewall above the boundary layer, which is consistent with the E86 assumption.

As further support for this conclusion, we show a plot of $s$ using $M$ as the horizontal coordinate in Fig. 5. In constructing this figure, we had to account for areas of 


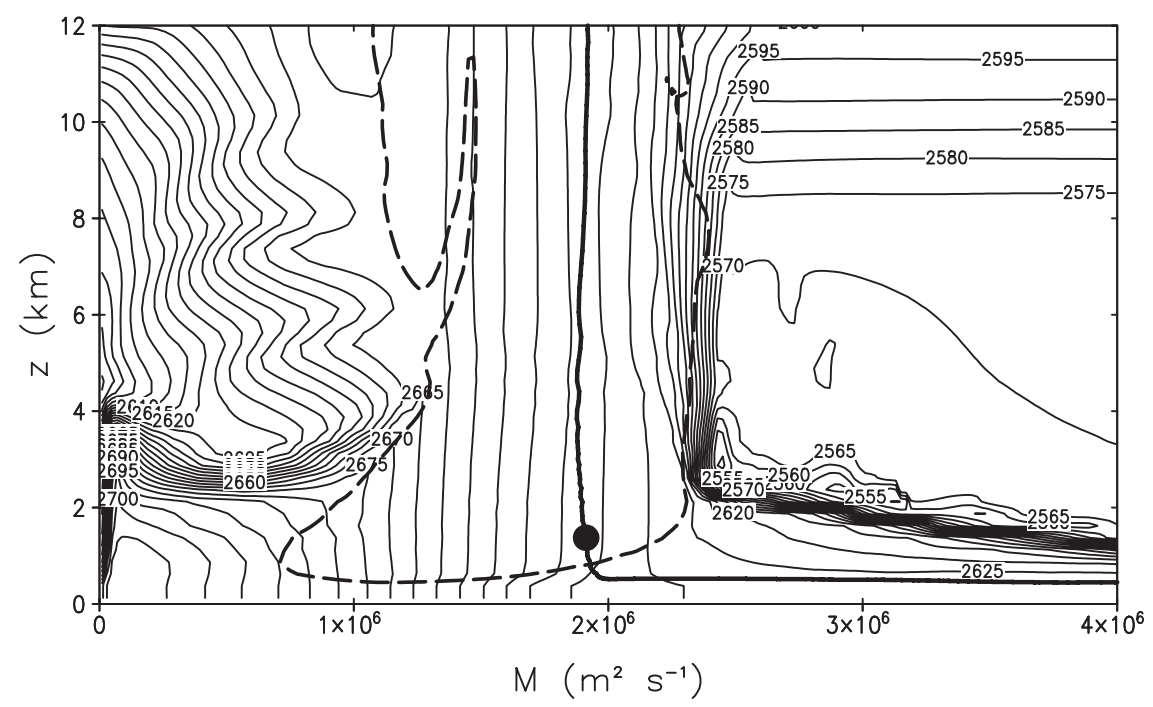

FIG. 5. As in Fig. 4a, but $s$ (with contour interval of $5 \mathrm{~J} \mathrm{~kg}^{-1} \mathrm{~K}^{-1}$ ) is plotted using $M$ as the horizontal coordinate (see text for details). The thick dashed contour denotes $w=2 \mathrm{~m} \mathrm{~s}^{-1}$, which approximately indicates the location of the eyewall.

inertial instability (enclosed by dashed contours in Fig. 4b) for which there are multiple locations having the same value of $M$ at a specified height. So, for Fig. 5, we use the value of $s$ from the $M$ surface at smallest $r$. This problem does not arise in the eyewall (for $z<10 \mathrm{~km}$ ), which is the region we are interested in analyzing, and hence does not affect the conclusions drawn here. Consistent with E86's assumption of moist slantwise neutrality, there is approximately one value of $s$ for a given value of $M$ in the eyewall (Fig. 5). Above the boundary layer in the eyewall, the maximum variation in $s$ along an $M$ surface is $\sim 2 \mathrm{~J} \mathrm{~kg}^{-1} \mathrm{~K}^{-1}$, which as discussed earlier is attributable to weak diffusion in the model.

Based on these analyses, we conclude that an approximate condition of moist slantwise neutrality is achievable in the eyewall of a numerical simulation. It follows that this component of E-PI is probably not the source of the discrepancy between $v_{g \text {,max }}$ and E-PI in the control simulation.

\section{b. PBL closure}

The PBL closure in E-PI can be expressed succinctly by (8) (with $\varepsilon=0$ if dissipative heating is not considered). Several simple assumptions are made in the derivation of this relation, and some of these assumptions may undermine the accuracy of the closure. For example, it has been assumed that radial advection balances vertical turbulent transport in the boundary layer for both $s$ and $M$. BR09b found that radial turbulent transport is an important process in some simulations, so its neglect in the E86 PBL formulation might be a problem. The E86 PBL model also assumes well-mixed profiles of $u, s$, and $M$, although it is clear that the boundary layer in the numerical simulations cannot be characterized as well mixed (e.g., Fig. 4, below $\sim 1$ km).

In our analysis, we choose not to evaluate the underlying assumptions in the derivation that yields (8). Rather, we evaluate directly the final closure, given by (8), because this relation is what the analytic model (10) ultimately relies upon. To this end, we evaluate the left side of this relation by dividing the radial gradient of $s$ by the radial gradient of $M$ at the top of the boundary layer (i.e., at the height of $v_{\max }$ ). For the right side of (8), we evaluate these terms directly using the corresponding code from the numerical model. Results from the control simulation (Fig. 6) suggest qualitatively good agreement between the two sides of this relation (except in the hurricane eye, where this relation is not needed). Specifically, the left side of (8) varies in the same way as the right side of (8). In the eyewall (shaded region of Fig. 6), the two terms are quantitatively different by $\sim 50 \%$, on average. However, we note that the right side of (8), which is ultimately used for E-PI, is larger in magnitude; this would introduce a positive bias, and hence cannot explain the tendency for E-PI to be less than $v_{g \text {,max }}$.

To check on the generality of these results, we conduct the same analysis on the BR09b simulations. We show results evaluated at the location of $v_{\max }$ in Fig. 7. To investigate a broad sample of hurricane structures and intensities, we show results from simulations using different values for the horizontal turbulence length scale $\left(l_{h}\right)$. To allow for comparison against numerical models that do not use dissipative heating (such as the model of RE87), we show results from simulations without dissipative 


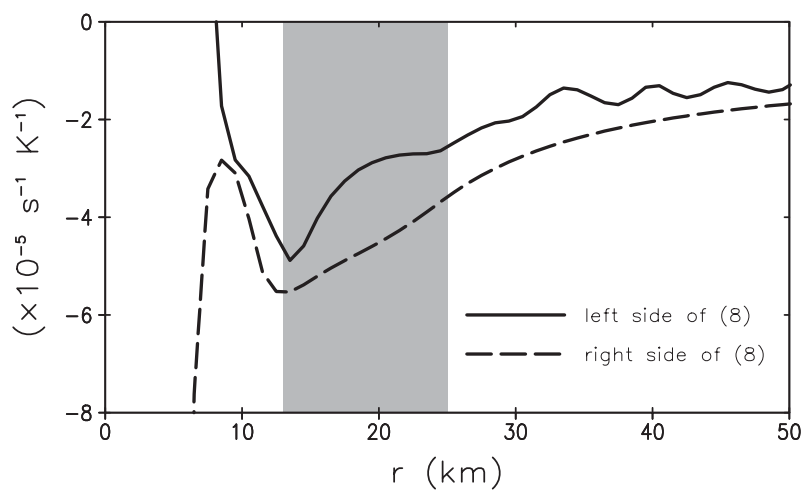

FIG. 6. Evaluation of (8) from the control simulation. The gray shaded region denotes the eyewall.

heating in gray and results from simulations with dissipative heating in black. We find that (8) is a sufficiently accurate relation for all of these simulations. In general, the right side of (8) tends to be slightly larger in magnitude than the left side. The quantitative agreement is somewhat better in these simulations (Fig. 7) than in the control simulation (Fig. 6); this difference might be attributable the larger value of $l_{v}$ in the BR09b simulations, which yields deeper (and thus better resolved) boundary layers.

Finally, we have also checked the assumption that the height of $v_{\max }\left(z_{m}\right)$ is roughly equivalent to the top of the boundary layer ( $h$, defined as the location where viscous effects become negligible). This assumption is needed to match the free-atmosphere component to the PBL closure in E-PI (see section 2b). Detailed analysis (not shown) supports this assumption, which can also be inferred from Figs. 4 and 5, in which $s$ and $M$ are approximately conserved along trajectories above $v_{\max }$ (dot in these two figures) but where trajectories cross contours of $s$ and $M$ below the location of $v_{\text {max }}$.

Based on these results, we conclude that the E86 PBL closure is accurate for its intended purpose, which, we reiterate, is to provide an estimate of $d s / d M$ at the top of the boundary layer. We also find that the PBL closure might be introducing a slight positive bias into the E-PI calculation because the right side of (8) (which is used in E-PI) tends to overestimate the left side of (8). This overestimation might be compensated by one of the other minor assumptions discussed in section 2 .

\section{c. Gradient-wind and hydrostatic balance}

We now evaluate the assumption of gradient-wind and hydrostatic balance (i.e., thermal wind balance). Using the governing equations from this numerical model, gradient-wind balance is expressed mathematically as

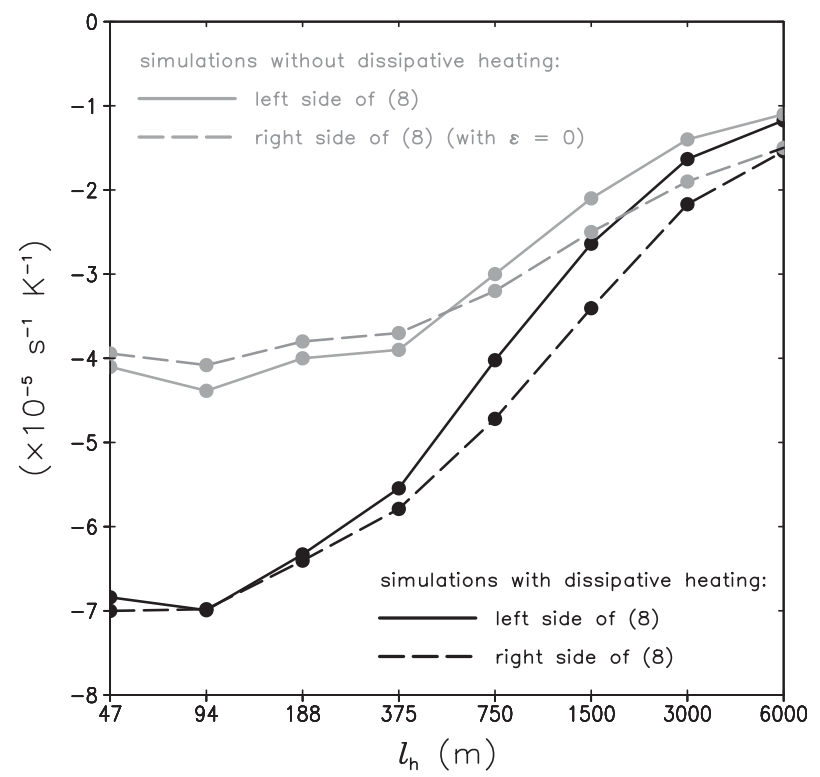

FIG. 7. Evaluation of (8) (at the location of $v_{\max }$ only) from the BR09b simulations. Black lines are from simulations with dissipative heating and gray lines are from simulations without dissipative heating.

$$
\underbrace{-c_{p} \theta_{v} \frac{\partial \pi}{\partial r}}_{U_{\mathrm{pg}}} \underbrace{+\frac{v^{2}}{r}}_{U_{\mathrm{ce}}} \underbrace{+f v}_{U_{\mathrm{Co}}}=0
$$

(the labels under each term are provided for convenience). Hydrostatic balance is expressed mathematically as

$$
\underbrace{-c_{p} \theta_{v} \frac{\partial \pi^{\prime}}{\partial z}}_{W_{\mathrm{pg}}} \underbrace{+B}_{W_{\mathrm{B}}}=0,
$$

where $\pi^{\prime}$ is perturbation $\pi$ and $B$ is buoyancy, as defined in BR09b.

Analysis of gradient-wind balance in the control simulation is shown in Fig. 8. The cyclone is in gradient-wind balance where the analysis in Fig. 8 is approximately zero. Although most regions of the free atmosphere are characterized by approximate gradient-wind balance, there is clearly not a balance in the eyewall. At the location of $v_{\max }$, the sum $U_{\text {ce }}+U_{\mathrm{Co}}$ is twice the magnitude of $U_{\mathrm{pg}}$. This imbalance implies a supergradient flow, in which the outward-directed force per unit mass (primarily from centrifugal acceleration) exceeds the inward-directed force per unit mass (from the pressure gradient).

Analysis of hydrostatic balance is shown in Fig. 9. The tropical cyclone is in hydrostatic balance where the analysis in Fig. 9 is approximately zero. In most locations, the flow is in approximate hydrostatic balance. The primary 


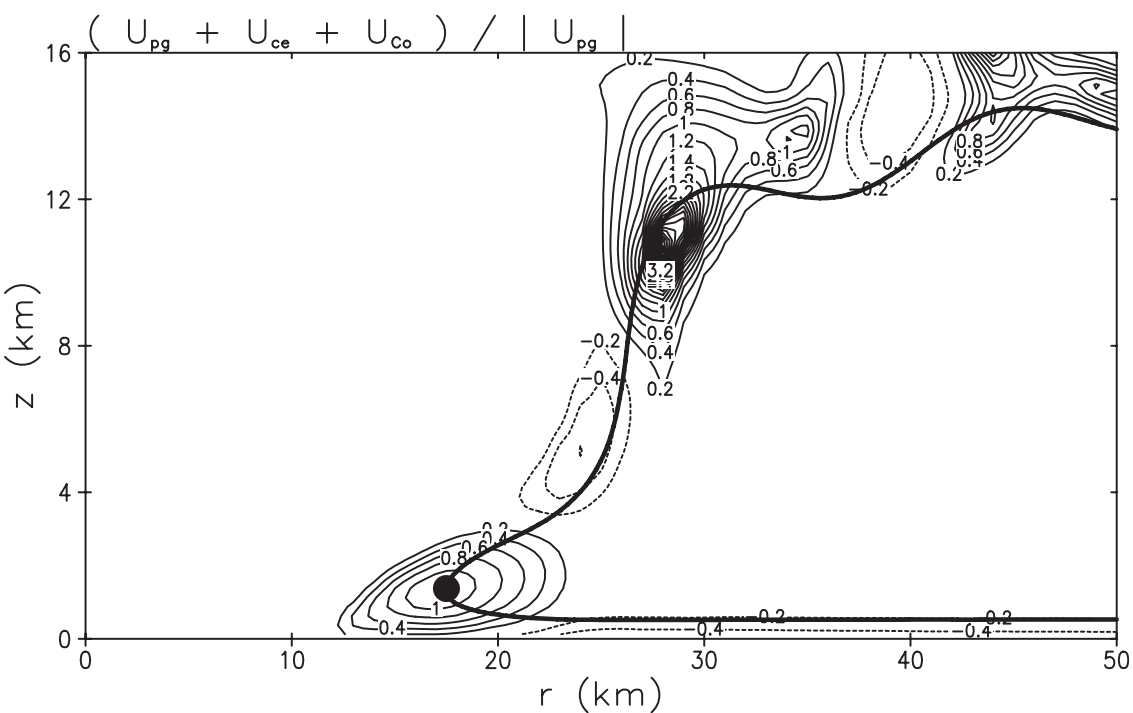

FIG. 8. Analysis of gradient-wind balance in the control simulation. Contoured is the sum $U_{\mathrm{pg}}+U_{\mathrm{ce}}+U_{\mathrm{Co}}$ normalized by the magnitude of $U_{\mathrm{pg}}$, with a contour interval of 0.2 . The zero contour is excluded. The trajectory that passes through $v_{\max }$ is illustrated by the thick line, and the dot denotes the location of $v_{\text {max }}$.

exception is the eyewall, where the magnitude of buoyancy exceeds the magnitude of the pressure gradient, often by $20 \%$, but in some places by more than a factor of 2 .

Analysis of the BR09b simulations (not shown) yields the same conclusions; that is, gradient-wind and hydrostatic balance are approximately met over most of the free atmosphere, but not in the eyewall. We find that the weaker tropical cyclones in these simulations (i.e., the more diffusive simulations that have larger $l_{h}$ ) are much closer to gradient-wind and hydrostatic balance, although all simulations have some degree of imbalance in the free atmosphere. As an example, for $l_{h}=3000 \mathrm{~m}$, the sum $U_{\text {ce }}+U_{\text {Co }}$ is $15 \%$ greater than the magnitude of $U_{\mathrm{pg}}$, and $W_{\mathrm{B}}$ is $5 \%$ greater than the magnitude of $W_{\mathrm{pg}}$.

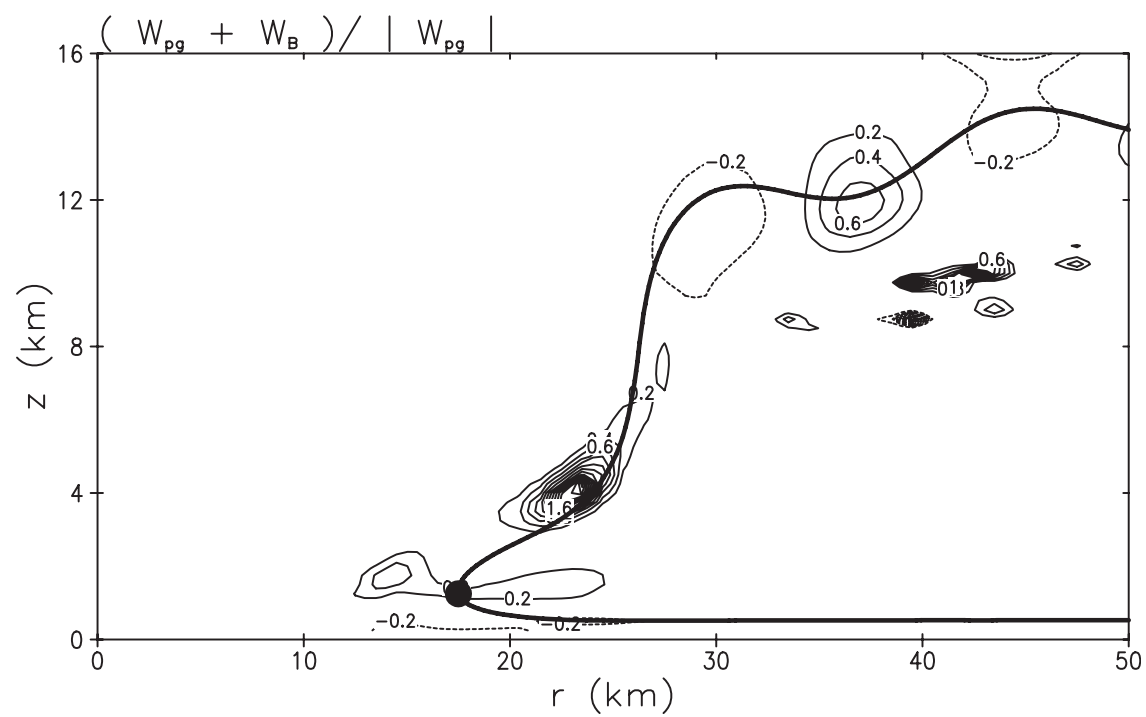

FIG. 9. Analysis of hydrostatic balance in the control simulation. Contoured is the sum $W_{\mathrm{pg}}+W_{\mathrm{B}}$ normalized by the magnitude of $W_{\mathrm{pg}}$, with a contour interval of 0.2 . The zero contour is excluded. The trajectory that passes through $v_{\max }$ is illustrated by the thick line, and the dot denotes the location of $v_{\max }$. 


\section{The impact of unbalanced flow}

Of the three primary components of E-PI, only the dynamical component - that is, the assumption of gradientwind and hydrostatic balance in the free atmosphere-is not applicable to the control simulation. In this section, we evaluate whether this component can be solely responsible for the difference between E-PI and numerically modeled intensity.

As discussed earlier, E-PI is a model for the maximum gradient wind speed. Thus, the existence of supergradient flow does not necessarily undermine the applicability of E-PI as a model for the underlying (i.e., background) balanced flow. We have already provided an estimate of this balanced flow $\left(v_{g}\right)$ in Fig. 3; the analysis shows $v_{g}>$ E-PI over a $20-\mathrm{km}$ region near the eyewall. This difference suggests that some process allows $v_{g}$ to exceed the maximum value predicted by E-PI.

Nevertheless, a different conclusion is reached by more careful analysis of the manner in which $v_{g}$ has been calculated for Fig. 3. We note that the pressure field from the model $(\pi)$ is used to calculate $v_{g}$ [see (11)]. No attempt has been made to separate the pressure field into a component associated with balanced flow and a component associated with unbalanced flow. To explain, consider the radial velocity equation,

$$
-\frac{\partial \phi}{\partial r}+\frac{v^{2}}{r}=\frac{d u}{d t}
$$

where $\phi$ is the pressure variable and where the Coriolis term has been neglected for simplicity. If the term on the right side is negligible, then (14) is the definition of gradient-wind balance. However, the right side of (14) is not negligible in the control simulation. Furthermore, a more complete analysis would decompose variables into components associated with a balanced background flow (denoted by subscript $g$ ) and a perturbation from that flow (denoted with superscript prime); that is,

$$
-\frac{\partial \phi_{g}}{\partial r}+\frac{v_{g}^{2}}{r}=\frac{\partial \phi^{\prime}}{\partial r}-\frac{v^{\prime 2}}{r}-\frac{2 v_{g} v^{\prime}}{r}+\frac{d u}{d t} .
$$

In this context, the method used for Fig. 3 is equivalent to moving the first term of the right side onto the left side (i.e., the full pressure field is used for Fig. 3), and then assuming that the remaining terms on the right side are negligible. Consequently, this method inherits some information about the unbalanced flow (via $\phi^{\prime}$ ), and the value of $v_{g}$ thus derived is not representative of a truly balanced flow.

As an example from a simpler flow in which the balanced background flow is clearer, Howells et al. (1988, their Fig. 9) show a pressure oscillation that coincides with an unbalanced oscillation in the flow. The pressure perturbation appears to oscillate by a factor of 2 . If an equation analogous to (11) were used to calculate $v_{g}$, then the result would similarly show an oscillation (in $v_{g}$ ) that would differ from the known balanced flow for their case.

Hence, we argue that $v_{g}$ calculated from (11) cannot be directly compared to E-PI (which has imposed gradientwind balance everywhere). Rather, $v_{g}$ calculated from (11) needs to be interpreted as the azimuthal velocity that would be necessary to balance the pressure field if all else remained equal (including retention of unbalanced flow).

As a simple ${ }^{4}$ yet insightful way around this dilemma, we utilize the analytic equations derived by E86, which impose gradient-wind balance everywhere in the free atmosphere (assuming moist slantwise neutrality). Most important for this purpose is E86's (11),

$$
\left.r^{2}\right|_{M_{g}}=-M_{g}\left[\frac{d s}{d M_{g}}\left(T-T_{\text {out }}\right)\right]^{-1},
$$

which is essentially an equation for the shape of $M_{g}$ surfaces in the free atmosphere. We have already shown that the eyewall of the control simulation is in a state of moist slantwise neutrality (section 4a). We can thus use (16) to calculate hypothetical trajectories for flow that was truly in gradient-wind balance.

To this end, we utilize conditions along actual trajectories from the control simulation-specifically, we use $T(z), M, d s / d M$, and $T_{\text {out }}$ and then we use (16) to compute $r$ for hypothetical trajectories. We use conditions along the actual trajectory that passed through $v_{\text {max }}$. Figure 10 shows a comparison of the hypothetical trajectory (gray line) with the actual trajectory (black line). There are two key differences in these trajectories: 1) the hypothetical trajectory is always located at larger $r$ and 2) there is no oscillation in the hypothetical trajectory, in the sense that it smoothly moves toward larger $r$ as $z$ increases.

The larger radius of the hypothetical trajectory is consistent with weaker intensity. Using the value of $M$ and the minimum radius along this trajectory (i.e., at $z=z_{m}$ ), we find a maximum gradient wind of $v_{g}=72 \mathrm{~m} \mathrm{~s}^{-1}$. This is the same value as E-PI determined using the right side of (10). Thus, the suggested discrepancy between $v_{g}$ and E-PI in Fig. 3 is actually attributable to the method used to calculate $v_{g}$ for this figure. We believe the analysis in Fig. 10 is more directly comparable to E-PI, and it suggests that E-PI does, in fact, provide an accurate upper bound for maximum balanced flow in simulated tropical cyclones.

\footnotetext{
${ }^{4}$ In principle, a more rigorous method might be able to use potential vorticity inversion to simultaneously retrieve both the mass and wind fields.
} 


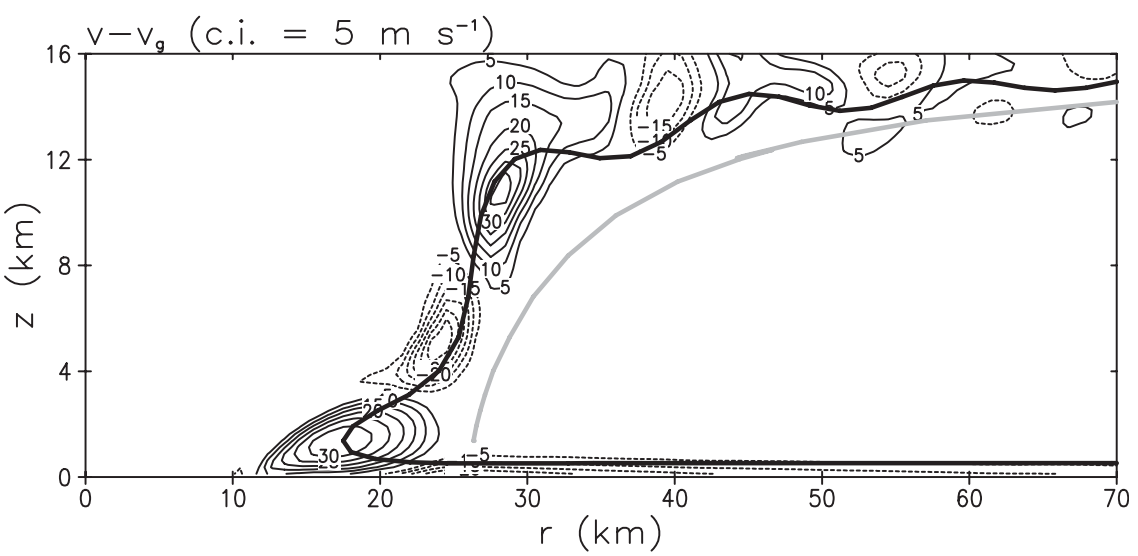

FIG. 10. Comparison of the trajectory that passes through $v_{\max }$ from the control simulation (thick black line) with a hypothetical trajectory having the same thermodynamic conditions but assuming gradient-wind and hydrostatic balance (gray line). Plotted for reference is $v-v_{g}$ (contours) with contour interval of $5 \mathrm{~m} \mathrm{~s}^{-1}$.

Concerning the different shapes of the two trajectories in Fig. 10, the differences reveal an oscillation in the actual parcel trajectory, whereby the actual parcel is accelerated toward the hypothetical trajectory, then away from the hypothetical trajectory, and so forth. The behavior along the actual trajectory is qualitatively similar to flows in classic analytic solutions for rotating-flow boundary layers, such as that presented by Bödewadt (1940), Rott and Lewellen (1966), and Kuo (1971). These studies similarly found flow that oscillates between subgradient and supergradient. The maximum inertial overshoot $\left(v / v_{g}\right)$ varies depending on the (specified) radial gradient of $v_{g}$ at the top of the boundary layer, with values between 1.27 and 1.6 reported by Rott and Lewellen (1966); the overshoot in our numerical simulation falls within this range.

From these results, we draw several important conclusions. First, E-PI should not be considered as an upper limit on $v$ because E-PI does not account for unbalanced flow, which can be significant in the eyewall of tropical cyclones (see also SMV08). Second, $v_{g}$ can exceed E-PI if, as in (11), $v_{g}$ is calculated using the full pressure field, because the latter contains pressure perturbations attributable to the unbalanced flow. Last, estimates of $v_{g}$ for a truly balanced background state do not exceed E-PI for this case; in fact, simulations with a numerical model that imposes dynamical balance (Emanuel 1989, 1995, 1997) support this conclusion.

\section{PI theory for unbalanced flow}

\section{a. Derivation}

For a more complete analysis of PI, consideration of unbalanced flow is clearly required. In this section, we present and then evaluate an analytical model that al- lows for gradient-wind and hydrostatic imbalance. The derivation is an extension of the technique used by D. K. Lilly to study tornadoes in the 1960s (Lilly 1969) and hurricanes in the early 1980s (D. Lilly, unpublished manuscript). A brief review of Lilly's work on hurricanes is summarized in Emanuel (2004). Lilly's intention was to derive analytic solutions for the flow field in tornadoes and hurricanes. Using conservation equations for mass, momentum, and entropy, he derived a single equation for streamfunction, which can then be solved given appropriate boundary conditions. This is the same technique used by Long (1953). Our derivation of the analogous Long's equation (see appendix B for details) yields the relation

$$
\begin{aligned}
-\frac{\eta}{\rho_{d} r}+\frac{d}{d \psi}\left(\frac{u^{2}}{2}+\frac{w^{2}}{2}\right)= & -\frac{d}{d \psi}\left(\frac{v^{2}}{2}+g z+c_{p} T+L_{0} q_{v}\right. \\
& \left.+\frac{f M}{2}\right)+\frac{1}{2 r^{2}} \frac{d M^{2}}{d \psi}+T \frac{d s}{d \psi},
\end{aligned}
$$

(see also Emanuel 2004, p. 170), where $\eta \equiv \partial u / \partial z-$ $\partial w / \partial r$ is the azimuthal vorticity, $\rho_{d}$ is the density of dry air, $\psi$ is the streamfunction (defined in appendix B), and $L_{0}$ is a constant latent heat of vaporization (from Bryan 2008). As pointed out by Lilly (see Emanuel 2004), if the flow is in gradient-wind and hydrostatic balance, then the left side of (17) would be exactly zero (see also appendix B).

To arrive at a solution for maximum intensity, (17) is integrated over a control volume encompassing the eyewall and the upper-level outflow of a tropical cyclone, as illustrated in Fig. 11. The same technique was used by BE98 (their section 3.2). Details of our derivation are provided in appendix $\mathrm{B}$. We find 


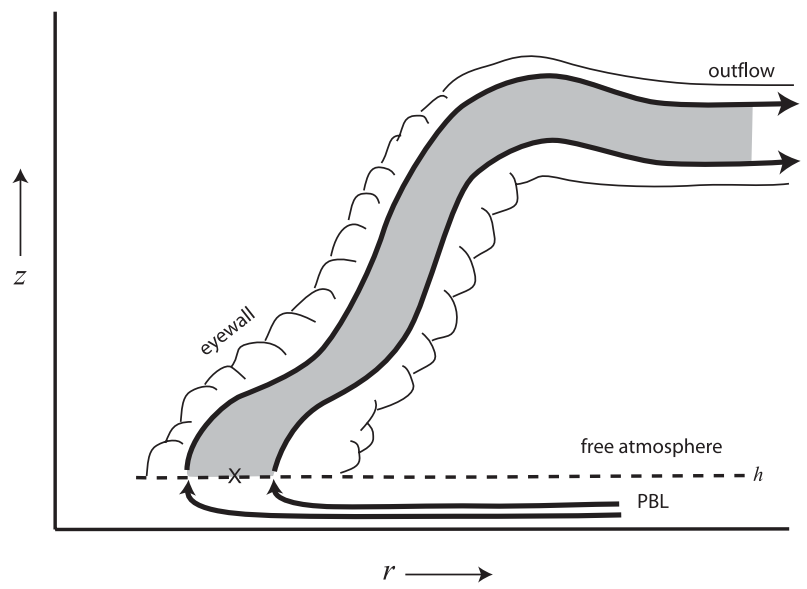

FIG. 11. A schematic illustrating the control volume (shaded region) used for the derivation of $\mathrm{PI}^{+}$. The control volume is bounded by two closely spaced streamlines (thick solid lines) in the eyewall. The variables $\psi, s, M$, and $E$ are constant along these trajectories in the free atmosphere (i.e., above the dashed line), assuming inviscid pseudoadiabatic flow. The " $\mathrm{X}$ " denotes the location of $v_{\max }$.

$$
\frac{1}{r_{b}^{2}}=\frac{1}{r_{0}^{2}}-2\left(T_{b}-T_{0}\right) \frac{d s}{d M^{2}}-2 \frac{\eta_{b}}{\rho_{b} r_{b}} \frac{d \psi}{d M^{2}}
$$

where subscript $b$ denotes evaluation at the top of the boundary layer and subscript 0 denotes evaluation at the outflow (at large $r$ ). By further assuming $r_{0} \gg r_{b}$ and $M_{b} \approx r_{b} v_{b}$, we obtain a relation for $v_{\text {max }}$ :

$$
\begin{aligned}
& v_{\max }^{2}=-\left(T_{b}-T_{0}\right) M_{b} \frac{d s}{d M}-\frac{M_{b} \eta_{b}}{\rho_{b} r_{b}} \frac{d \psi}{d M} \text { at } \\
& (r, z)=\left(r_{\max }, z_{\max }\right) .
\end{aligned}
$$

The first term on the right side of (19) has the same form as the free-atmosphere component of E-PI [see (7)]. In fact, if gradient-wind and hydrostatic balance had been assumed, then the last term of (19) would have been exactly zero and this relation would be equivalent to (7). Thus, E-PI as reviewed in section 2 may be viewed as a special case within this framework.

Both Lilly and BE98 ultimately neglected the unbalanced flow (i.e., inertial) term. Evaluation of this term is difficult because some variables (especially $\psi$ ) are difficult to obtain from observations and numerical simulations. We thus modify this term by utilizing the definition of $\psi$, by again assuming $M_{b} \approx r_{b} v_{b}$, and by making use of the fact that $\partial v_{b} / \partial r=0$ at the location of maximum winds; this yields

$$
\begin{aligned}
& v_{\text {max }}^{2}=-\left(T_{b}-T_{0}\right) M_{b} \frac{d s}{d M}+r_{b} \eta_{b} w_{b} \text { at } \\
& (r, z)=\left(r_{\text {max }}, z_{\text {max }}\right) .
\end{aligned}
$$

In this form, the term accounting for unbalanced flow (the last term) contains variables that are more intuitive and are more easily calculated from numerical model output and observations. To help interpret this term, we note that the radial velocity equation, (B1), can be expressed as

$$
\frac{d u}{d t} \equiv u \frac{\partial u}{\partial r}+\eta w+w \frac{\partial w}{\partial r}=\left(\frac{v^{2}}{r}-\frac{\hat{v}_{g}^{2}}{r}\right),
$$

where $\hat{v}_{g}^{2} / r \equiv \alpha_{d} \partial p / \partial r$, and the Coriolis term is neglected because it is small at the location of $v_{\max }$. Numerical simulations and observations often show that $u \approx 0$ at the location of $v_{\max }$ and that $w$ is a maximum here also, so that $\partial w / \partial r \approx 0$. Thus, (21) can be reduced to

$$
\eta w \approx\left(\frac{v^{2}}{r}-\frac{\hat{v}_{g}^{2}}{r}\right) .
$$

In a supergradient flow, the right side of (22) is greater than zero, and thus $\eta w$ is necessarily positive. Hence, the final term in (20) is always a positive contribution to $v_{\max }$ in supergradient flows. Moreover, simple calculations based on the classic Bödewadt rotating-flow boundary layer solution [see Schlichting (1979, p. 228)] show essentially the same balance of terms. To summarize, the inertial terms in the momentum equations are not negligible in supergradient flows, and the analyses above demonstrate that they contribute to an increase in $v_{\max }$ that is proportional to $\eta w$ at the location of $v_{\max }$.

Our derivation has so far considered only the free atmosphere. A boundary layer can be incorporated in the same manner as in section $2 b .^{5}$ Repeating those steps, using the same approximations, we find

$$
\begin{aligned}
& v_{\max }^{2}=\alpha\left[\frac{C_{E}}{C_{D}}\left(T_{b}-T_{0}\right)\left(s_{\text {surf }}-s_{0}\right)+r_{b} \eta_{b} w_{b}\right] \text { at } \\
& (r, z)=\left(r_{\text {max }}, z_{\text {max }}\right) .
\end{aligned}
$$

As it will be convenient to compare this solution to E-PI, we note that (23) can be expressed as follows:

$$
v_{\max }^{2}=(\mathrm{E}-\mathrm{PI})^{2}+\gamma,
$$

where (E-PI) ${ }^{2}$ is given by the right side of (10), $\gamma \equiv$ $\alpha r_{b} \eta_{b} w_{b}$, and all terms are evaluated at the location of maximum tangential velocity.

\footnotetext{
"In this section, the definitions of "free atmosphere" and "boundary layer" are the same as in section 2 and are distinguished primarily by the importance of viscous terms.
} 
The " $\gamma$ " term was derived in essentially the same manner by BE98 [see the final term in brackets in their (33); our relation can be derived from BE98's (33) by using the PBL assumptions and approximations from our section $2 \mathrm{~b}$ and by recognizing that the budget of $M$ in the boundary layer can be expressed as $\psi \partial M / \partial r=$ $\left.-\rho r^{2} C_{D}|V| v_{0}\right]$. They concluded that this term is "roughly three orders-of-magnitude smaller than the other terms and thus may be neglected" (p. 237). We re-evaluate their conclusion using numerical model output and observations in the next two subsections.

\section{b. Evaluation using model output}

We evaluate (24) from numerical model output using the same techniques as before. We refer to PI calculated using the entire right side of $(24)$ as $\mathrm{PI}^{+}$. We find $\mathrm{PI}^{+}=$ $107 \mathrm{~m} \mathrm{~s}^{-1}$ in the control simulation, which is comparable to $v_{\max }\left(=109 \mathrm{~m} \mathrm{~s}^{-1}\right)$. The close correspondence between $v_{\max }$ and $\mathrm{PI}^{+}$supports our argument (section 5) that the neglect of unbalanced flow effects is mostly responsible for the systematic underprediction by E-PI.

From the model output we find $r_{b}=17.5 \mathrm{~km}, \eta_{b}=$ $0.03 \mathrm{~s}^{-1}, w_{b}=8 \mathrm{~m} \mathrm{~s}^{-1}$, and $\alpha=1.5$, for which $\gamma=$ $6300 \mathrm{~m}^{2} \mathrm{~s}^{-2}$. Because E-PI $=72 \mathrm{~m} \mathrm{~s}^{-1}$, and thus $(\mathrm{E}-\mathrm{PI})^{2}=5200 \mathrm{~m}^{2} \mathrm{~s}^{-2}$, the effects of unbalanced flow contribute as much to maximum intensity as balanced flow for this case. Clearly, the unbalanced flow term is not negligible for this simulation.

We surmise that BE98 found the unbalanced-flow term to be negligible (compared to the balanced-flow term) because they were probably using a more diffusive numerical model. We have previously shown (in BR09b) that simulated tropical cyclones are closer to gradientwind balance as radial turbulence increases. To elaborate, we show in Fig. 12 an evaluation of $v_{\max }$, E-PI, and $\mathrm{PI}^{+}$from the BR09b simulations in which $l_{h}$ is varied. For $l_{h} \geq 3000 \mathrm{~m}$, the contribution from unbalanced flow is negligible; for $l_{h}<3000 \mathrm{~m}$, the effects of unbalanced flow on maximum intensity are important. In the context of (24), stronger radial turbulence must weaken $\gamma$ by reducing the amplitude of both $\eta_{b}$ and $w_{b}$, which we confirm by inspection of model output (not shown). The radius of maximum winds $\left(r_{b}\right)$ actually increases with stronger turbulence in our model simulations, but apparently this is not great enough to counter the reductions in $\eta_{b}$ and $w_{b}$.

In an evaluation of E-PI, RE87 conducted numerical simulations using $l_{h}=3000 \mathrm{~m}$; consistent with their conclusions, we find that E-PI is a good predictor of maximum intensity for this model setup (Fig. 12). In a later study, PM03 used $l_{h}=750 \mathrm{~m}$ in their " $4 \mathrm{x}$ " simulation, and they found a large difference between $v_{\max }$ and E-PI; our analysis shows that this difference is en-

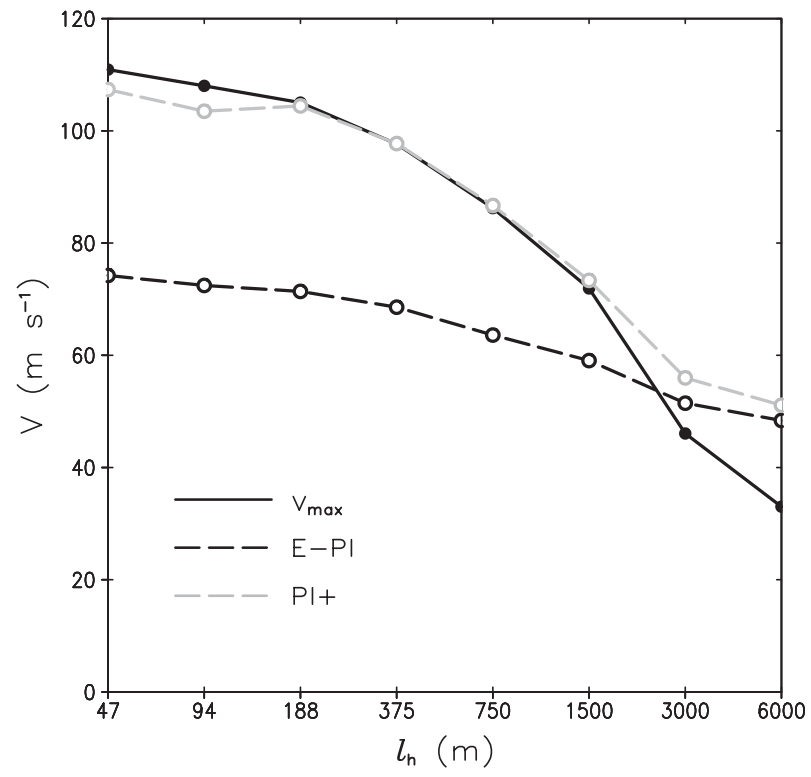

FIG. 12. Maximum azimuthal velocity ( $v_{\max }$, solid), E-PI (black dashed), and $\mathrm{PI}^{+}$(gray dashed) for the BR09b simulations.

tirely attributable to the assumption of gradient-wind balance in E-PI (Fig. 12).

\section{c. Evaluation using observations}

The $\gamma$ term in (24) can be conveniently evaluated using appropriate observations of steady axisymmetric hurricanes. As an example, we utilize the analyses of Hurricane Isabel (2003) from Bell and Montgomery (2008). In Table 1 , we list estimates of $v_{\max }$, E-PI, and $r_{b}$ as determined by Bell and Montgomery (2008) (see their article for details and uncertainties). From their Fig. 4, we estimate $w_{b}$ and $\eta_{b}$ (both of which are evaluated at the location of $\left.v_{\max }\right)$. We note that $u \approx 0$ at the location of $v_{\max }$ in their analyses, and thus assumptions about the geometry of the flow inherent in (24) are valid. From other analyses in their article we find $\alpha \approx 1.4$ on all days. Using (24), we find $\mathrm{PI}^{+}$to be $5 \%-30 \%$ higher than E-PI (Table 1). For all days, $\mathrm{PI}^{+}$is actually less than $v_{\max }$. The largest difference is on 12 September, but this may be attributable to the unsteady evolution (probably an eyewall replacement cycle) on this day (Bell and Montgomery 2008, p. 2030). For all days, the discrepancy may be attributable to uncertainties in the analyses. We suspect that the values of $w_{b}$ are most uncertain, and we note that they are 5-10 $\mathrm{m} \mathrm{s}^{-1}$ less than values of $w_{b}$ from our numerical simulations and from some direct observations during aircraft penetrations of intense tropical cyclones (e.g., Marks et al. 2008). Furthermore, if we accept the values of $v_{\max }$ and E-PI $\left(\equiv \hat{v}_{g}\right)$ as accurate, then (22) suggests that $\eta_{b} w_{b}$ must be $\sim 2-9$ times larger 
TABLE 1. Estimates of $\mathrm{PI}^{+}$using the observational analyses of Bell and Montgomery (2008). See text for details.

\begin{tabular}{lcccccc}
\hline \hline Date & $v_{\max }\left(\mathrm{m} \mathrm{s}^{-1}\right)$ & E-PI $\left(\mathrm{m} \mathrm{s}^{-1}\right)$ & $r_{b}(\mathrm{~km})$ & $w_{b}\left(\mathrm{~m} \mathrm{~s}^{-1}\right)$ & $\eta_{b}\left(\mathrm{~s}^{-1}\right)$ & $\mathrm{PI}^{+}\left(\mathrm{m} \mathrm{s}^{-1}\right)$ \\
\hline 12 Sep & 80 & 61 & 25 & 2 & 0.006 & 64 \\
13 Sep & 76 & 57 & 45 & 3 & 0.012 & 74 \\
14 Sep & 74 & 59 & 50 & 2 & 0.008 \\
\hline
\end{tabular}

than the values suggested by Table 1 . Despite these uncertainties in the observations, we are nevertheless able to conclude that unbalanced flow effects are not negligible in some natural tropical cyclones and that they contribute significantly to maximum intensity.

\section{Discussion}

We now compare our findings to those from similar studies (see also section 1 for a brief review). PM03 concluded that their simulated hurricane was not in a state of moist slantwise neutrality, whereas we conclude that our simulated hurricane does meet this condition. Evidence suggests that PM03 used a mathematical formulation of entropy (from RE87) that is not appropriate for their simulations, ${ }^{6}$ which gives the appearance that entropy is not conserved along $M$ surfaces. The consequences of different choices for defining entropy were explained in an analysis by BR09b (their section 3d). In their simulation that used a $7 \mathrm{~m} \mathrm{~s}^{-1}$ fall velocity (the same setup as PM03), they showed that one formulation of entropy suggests a decrease in entropy along trajectories, whereas a different formulation of entropy suggests an increase in entropy along trajectories (see Fig. 8 in BR09b). Our analysis herein uses a formulation for entropy that is consistent with the numerical model's governing equations and setup (i.e., pseudoadiabatic thermodynamics). We surmise that a more appropriate formulation of entropy applied to PM03's simulation would show an approximate state of moist slantwise neutrality in their simulation.

On the theoretical side, the present diagnosis of numerical simulation results agrees with the conclusion of SMV08 (555-556), finding that E-PI can be exceeded by supergradient winds in the boundary layer. It is shown here, moreover, that there is also gradient-wind imbalance in the eyewall above the boundary layer extending to the tropopause.

The present study (and the critique of the E86 model given in SMV08) is narrowly focused on the assumptions underlying the E86 hurricane model. We offer here in

\footnotetext{
${ }^{6}$ Appropriate formulations of moist entropy for the RE87 model can be found in Persing and Montgomery (2005) and Bryan and Rotunno (2009a).
}

conclusion our view of the E86 model in a broader context of analytical hurricane models through history. The earliest ideas (from the 1950s) conceived of the mature tropical cyclone as an idealized axisymmetric steady-state vortex (i.e., a primary flow) fundamentally influenced by momentum and heat exchange with the underlying surface through a flow in the radial-vertical plane [i.e., a secondary flow; see the review by Ooyama (1982, 370-371)]. In the context of engineering fluid mechanics, the influence of a lower stress-exerting surface on an axisymmetric steady-state vortex in a constant-density fluid had been studied for some time [see the review by Rott and Lewellen (1966)]. A central feature of such flows is the strong secondary flows engendered by the inward-directed outer pressure gradient (associated with the outer vortex) acting in the boundary layer where the centrifugal force is reduced as a consequence of surface friction. A number of papers appeared in the 1970s bringing the techniques of engineering fluid dynamics to bear on hurricanes. For example, Carrier et al. (1971), among others, computed the boundary layers and the implied secondary flows for prescribed (constant density) primary flows meant to represent the mature hurricane. Ooyama (1982, p. 371) trenchantly characterized this work as being "... off the line of progress ..." because it severs the link between the outer-flow pressure distribution (which is considered a "given" in the boundary layer calculations) and the thermodynamic transformations that sustain it in real hurricanes. The outline of an analytical theory uniting dynamics and energetics was foreshadowed in Eliassen and Kleinschmidt (1957, 131-132) where it was recognized that an analytical theory for the hurricane must account for mass-wind balance in the primary flow, heat and momentum transfer at the sea surface, and the moist thermodynamics of air in the inup-and-out secondary circulation (see their Fig. 30). The first successful execution of such a model was done in E86 by including a primary-flow dynamics that includes buoyancy effects and secondary-flow dynamics that exploits the congruence of entropy $(s)$ and angular momentum $(M)$ surfaces in the outer flow and a simple model for relation of $s$ to $M$ in the boundary layer.

Despite the deficiencies of E86's model that have since been identified by PM03, SMV08, and the analyses herein, it has nevertheless been shown to be a reasonable upper limit for the vast majority of observed tropical 
cyclones (e.g., Emanuel 2000). One might then ask: how can E-PI do so well most of the time? According to our analysis, the answer is that E-PI makes not one but two assumptions that are not wholly applicable to natural tropical cyclones: 1) gradient-wind balance (as shown by SMV08) and 2) inviscid flow in the radial direction (as shown herein and by BR09b). These assumptions have offsetting effects: the assumption of gradient-wind balance, by itself, would make tropical cyclones too weak, and the assumption of inviscid flow, by itself, would make tropical cyclones too strong. The latter problem seems to be at the root of some numerical models' tendency to make tropical cyclones too intense, as discussed further by BR09b and Chen et al. (2008). Observed cyclones, as well as simulations with stronger radial diffusion, are more nearly in gradient-wind balance and the discrepancy between E-PI and numerical simulation results is reduced (Fig. 12).

Our overall view is that the E86 model satisfies the minimum requirements of a theoretical hurricane model because it unites dynamics and energetics, and the PI derived from that model is quantitatively sufficient most of the time (e.g., Emanuel 2000). Refining theoretical PI by estimating the supergradient winds in the boundary layer (as advocated by SMV08) and by including viscous effects in the radial direction (as advocated herein) is certainly required, but we have been as yet unable to find a straightforward way to include these effects in an a priori form of PI (i.e., one that uses only environmental conditions as input). Further study using numerical models, theory, and/or observations of intense tropical cyclones will be needed to set bounds on the likely enhancement of PI from unbalanced flow and the likely reduction from radial turbulent diffusion.

\section{Summary}

In this paper we evaluate the analytic model for maximum hurricane intensity that was derived by Emanuel (1986)-E-PI—with the goal of clarifying why E-PI underpredicts maximum hurricane intensity in some numerical model simulations. In our review of E-PI, we identify three primary components of this model: a dynamical component (the assumption of gradient-wind and hydrostatic balance in the free atmosphere), a thermodynamical component (reversible or pseudoadiabatic thermodynamics, although the pseudoadiabatic assumption yields higher intensities), and a boundary layer closure (which relates radial gradients of $s$ and $M$ at the top of the boundary layer to fluxes and stresses at the ocean surface). Using output from an axisymmetric numerical model in which E-PI is much less than simulated intensity, we find the thermodynamical component and the boundary layer closure to be accurate for our simulations. However, the dynamical component is clearly violated, consistent with the critique of E-PI by SMV08 (although herein we find imbalance in the eyewall from the surface to the tropopause). Further consideration of methods used to calculate gradient-wind speed $\left(v_{g}\right)$ from model output reveals that E-PI does not exceed $v_{g}$ of a truly balanced reference state, although estimates of $v_{g}$ can exceed E-PI if the impacts of unbalanced flow are not removed from the calculation of $v_{g}$. We thus argue that the only significant shortcoming of E-PI, as applied to our axisymmetric model simulations, is the assumption of balanced flow. To test this conclusion, we consider unbalanced flow effects in an analytic model of maximum intensity, and we find this more complete model to be an accurate descriptor of our simulations. Evaluation against an observed hurricane further supports the conclusion that unbalanced flow effects have a nonnegligible effect on intensity in some cases. Although our control simulation has minimal radial diffusion (consistent with fundamental assumptions of the E-PI hurricane model), we find that stronger radial diffusion damps the unbalanced flow effects, and this explains why some previous modeling studies (e.g., RE87 and BE98) concluded that E-PI was an accurate descriptor of maximum intensity in their cases. Some evidence suggests that natural tropical cyclones have nonnegligible turbulent diffusion in the radial direction (see section 3b of BR09b). Thus, as applied to natural hurricanes and some model simulations, E-PI actually has two shortcomings: the assumptions of gradient-wind balance and inviscid flow (particularly for the radial direction). Because these two processes have opposite effects on intensity, E-PI turns out to be a fairly reasonable upper limit for most tropical cyclones, as shown by Emanuel (2000). Nevertheless, a more complete PI model for tropical cyclones would need to account for gradient-wind imbalance and radial turbulent fluxes.

Acknowledgments. Helpful reviews of this manuscript were provided by Drs. Chris Davis, Kerry Emanuel, and Mike Montgomery.

\section{APPENDIX A}

\section{Maxwell Relation for Pseudoadiabatic Thermodynamics}

Exact expressions for pseudoadiabatic thermodynamics, such as that presented by Iribarne and Godson (1981, p. 143), usually contain complicated integral terms that are costly to evaluate numerically and are intractable 
for analytic studies. Thus, approximate expressions are typically used. To this end, Bryan (2008) presented a set of approximate thermodynamical expressions that reproduce pseudoadiabatic processes very well. The first law of thermodynamics can be expressed in terms of entropy as

$$
T d s=c_{p} d T+L_{0} d q_{v}-\alpha_{d} d p,
$$

where $L_{0}=2.555 \times 10^{6} \mathrm{~J} \mathrm{~kg}^{-1}$ is a constant value of the latent heat of vaporization. Total moist enthalpy $(h)$ in this system is governed by

$$
d h=c_{p} d T+L_{0} d q_{v}
$$

From (A1) and (A2) it follows that

$$
d h=T d s+\alpha_{d} d p,
$$

and thus

$$
\begin{aligned}
& \left(\frac{\partial h}{\partial p}\right)_{s}=\alpha_{d} \quad \text { and } \\
& \left(\frac{\partial h}{\partial s}\right)_{p}=T .
\end{aligned}
$$

Cross differentiation of (A4) yields

$$
\left(\frac{\partial \alpha_{d}}{\partial s}\right)_{p}=\left(\frac{\partial T}{\partial p}\right)_{s}
$$

which we utilize in section $2 \mathrm{a}$.

To put this relation in historical context, we note that (A5) is similar in form to the Maxwell relation in appendix 1 of E86. However, our relation uses the specific volume of dry air (whereas the specific volume in E86 is not clearly defined), and our solution is clearly applicable to pseudoadiabatic thermodynamics (whereas the thermodynamic assumption in E86 is not clear; it is stated to be reversible thermodynamics, but the correctand clearly different - set of Maxwell equations for reversible thermodynamics was provided by E88). We also note that one of the Maxwell relations for reversible thermodynamics [Eq. (8) in E88] is similar in form to (A5), but it is not an equivalent expression; most notably, total water mixing ratio must be held constant in the reversible case whereas no such constraint is required for (A5), and we also note that the mathematical formulations for $s$ are very different in the two systems [cf. (1) and (11) in Bryan (2008)].

\section{APPENDIX B}

\section{Details of Derivation for Unbalanced Flow}

Assuming steady, inviscid flow, and neglecting the mass of water vapor in the momentum equations (as in section $2 \mathrm{a}$ ), the governing equations for radial and vertical velocity are

$$
\begin{aligned}
& u \frac{\partial u}{\partial r}+w \frac{\partial u}{\partial z}=\frac{v^{2}}{r}+f v-\alpha_{d} \frac{\partial p}{\partial r} \quad \text { and } \\
& u \frac{\partial w}{\partial r}+w \frac{\partial w}{\partial z}=-g-\alpha_{d} \frac{\partial p}{\partial z} .
\end{aligned}
$$

Throughout this appendix, terms associated with unbalanced flow (i.e., the inertial terms) are placed on the left side. Using $\eta \equiv \partial u / \partial z-\partial w / \partial r, M \equiv r v+f r^{2} / 2$, and a streamfunction defined by $\rho_{d} r u=\partial \psi / \partial z$ and $\rho_{d} r w=$ $-\partial \psi / \partial r,(\mathrm{~B} 1)$ and (B2) can then be combined into one equation:

$$
\begin{aligned}
-\frac{\eta}{\rho_{d} r}+\frac{d}{d \psi}\left(\frac{u^{2}}{2}+\frac{w^{2}}{2}\right)= & -\frac{d}{d \psi}\left(\frac{v^{2}}{2}+g z\right) \\
& +\frac{v d M}{r} \frac{d M}{d \psi}-\alpha_{d} \frac{d p}{d \psi} .
\end{aligned}
$$

The first law of thermodynamics for pseudoadiabatic flow (A1) is used to eliminate pressure from (B3). Further rearrangement yields

$$
\begin{aligned}
-\frac{\eta}{\rho_{d} r}+\frac{d}{d \psi}\left(\frac{u^{2}}{2}+\frac{w^{2}}{2}\right)= & -\frac{d}{d \psi}\left(\frac{v^{2}}{2}+g z+c_{p} T+L_{0} q_{v}\right. \\
& \left.+\frac{f M}{2}\right)+\frac{1}{2 r^{2}} \frac{d M^{2}}{d \psi}+T \frac{d s}{d \psi}
\end{aligned}
$$

which is given in section 6 as (17).

Herein, we assume pseudoadiabatic thermodynamics, and we neglect the mass of water vapor from the momentum equations (as in section $2 \mathrm{a}$ ). It should be possible to instead use reversible thermodynamics, which would obviate the need to approximate the momentum equations. We note that such a derivation would yield an additional term that is proportional to $d Q / d \psi$. Emanuel (2004) stated that reversible thermodynamics was used in his derivation, although it appears that he used the same approximations as E86, which ultimately yields the identical result, (B4).

At this point, D. Lilly (unpublished manuscript) assumed gradient-wind and hydrostatic balance, for which the left side of (B4) is exactly zero. We do not make this assumption here. 
We note that a governing equation for total energy can be formed from (B1), (B2), (A1), a governing equation for azimuthal velocity $(d M / d t=0)$, and a governing equation for total moist entropy $(d s / d t=0)$. (Note that herein $d / d t \equiv u \partial / \partial r+w \partial / \partial z$.) We find $d E / d t=0$, where $E \equiv u^{2} / 2+v^{2} / 2+w^{2} / 2+g z+c_{p} T+L_{0} q_{v}$. The second term on the left side of (B4) can thus be combined with the first term on the right side of (B4):

$$
-\frac{\eta}{\rho_{d} r}+\frac{d E}{d \psi}=-\frac{d}{d \psi}\left(\frac{f M}{2}\right)+\frac{1}{2 r^{2}} \frac{d M^{2}}{d \psi}+T \frac{d s}{d \psi} .
$$

We place $d E / d \psi$ on the left side because it contains some terms that are attributable to unbalanced flow.

To arrive at a solution for maximum intensity, (B5) is integrated over a control volume (Fig. 11) that is enclosed by two closely spaced streamlines in the free atmosphere. Because we assume that viscous effects are negligible in the free atmosphere (i.e., above dashed line in Fig. 11), then $\psi, s, M$, and $E$ are conserved along these streamlines. The second term on the lhs and the first term on the rhs of (B5) vanish upon integration over the control volume. The remaining terms can be expressed as follows:

$$
-\left(\frac{\eta_{b}}{\rho_{b} r_{b}}-\frac{\eta_{0}}{\rho_{0} r_{0}}\right)=\frac{1}{2}\left(\frac{1}{r_{b}^{2}}-\frac{1}{r_{0}^{2}}\right) \frac{d M^{2}}{d \psi}+\left(T_{b}-T_{0}\right) \frac{d s}{d \psi},
$$

where subscripts denote an average value along the section of the control volume that crosses streamlines in the outflow (0) and at top of the boundary layer $(b)$ (see Fig. 11). The second term in parentheses on the left side is negligible compared to the first term. Further rearrangement yields (18) of section 6 . We note that the only term associated with unbalanced flow is the one with $\eta_{b}$.

\section{REFERENCES}

Bell, M. M., and M. T. Montgomery, 2008: Observed structure, evolution, and potential intensity of category 5 Hurricane Isabel (2003) from 12 to 14 September. Mon. Wea. Rev., 136, 2023-2046.

Bister, M., and K. A. Emanuel, 1998: Dissipative heating and hurricane intensity. Meteor. Atmos. Phys., 65, 233-240.

Bödewadt, U. T., 1940: Die Drehströmung über festem Grunde. Z. Agnew. Math. Mech., 20, 241-253.

Bryan, G. H., 2008: On the computation of pseudoadiabatic entropy and equivalent potential temperature. Mon. Wea. Rev., 136, 5239-5245.

_ entropy air in hurricane eyes on maximum hurricane intensity. J. Atmos. Sci., 66, 148-158.

_ and - 2009b: The maximum intensity of tropical cyclones in axisymmetric numerical model simulations. Mon. Wea. Rev., 137, 1770-1789.

Carrier, G. F., A. L. Hammond, and O. D. George, 1971: A model of the mature hurricane. J. Fluid Mech., 47, 145-170.
Chen, Y., R. Rotunno, W. Wang, C. Davis, J. Dudhia, and G. J. Holland, 2008: Large eddy simulations of an idealized hurricane. Preprints, Ninth Annual WRF Users' Workshop, Boulder, CO, NCAR, 9.2. [Available online at http://www.mmm.ucar. edu/wrf/users/workshops/WS2008/abstracts/9-02.pdf.]

Cione, J. J., P. G. Black, and S. H. Houston, 2000: Surface observations in the hurricane environment. Mon. Wea. Rev., 128, 1550-1561.

Cram, T. A., J. Persing, M. T. Montgomery, and S. A. Braun, 2007: A Lagrangian trajectory view on transport and mixing processes between the eye, eyewall, and environment using a highresolution simulation of Hurricane Bonnie (1998). J. Atmos. Sci., 64, 1835-1856.

Eliassen, A., and E. Kleinschmidt Jr., 1957: Dynamic Meteorology. Vol. 47, Handbuch der Physik, Springer-Verlag, 154 pp.

Emanuel, K. A., 1986: An air-sea interaction theory for tropical cyclones. Part I: Steady-state maintenance. J. Atmos. Sci., 43, 585-605.

_ 1988: The maximum intensity of hurricanes. J. Atmos. Sci., 45, $1143-1155$

, 1989: The finite-amplitude nature of tropical cyclogenesis. J. Atmos. Sci., 46, 3431-3456.

1995: Sensitivity of tropical cyclones to surface exchange coefficients and a revised steady-state model incorporating eye dynamics. J. Atmos. Sci., 52, 3969-3976.

_ 1997: Some aspects of hurricane inner-core dynamics and energetics. J. Atmos. Sci., 54, 1014-1026.

2000: A statistical analysis of tropical cyclone intensity. Mon. Wea. Rev., 128, 1139-1152.

_- 2004: Tropical cyclone energetics and structure. Atmospheric Turbulence and Mesoscale Meteorology, E. Fedorovich, R. Rotunno, and B. Stevens, Eds., Cambridge University Press, 165-191.

Hausman, S. A., K. V. Ooyama, and W. H. Schubert, 2006: Potential vorticity structure of simulated hurricanes. J. Atmos. Sci., 63, 87-108.

Howells, P. A., R. Rotunno, and R. K. Smith, 1988: A comparative study of atmospheric and laboratory-analogue numerical tornado-vortex models. Quart. J. Roy. Meteor. Soc., 114, $801-822$.

Iribarne, J. V., and W. L. Godson, 1981: Atmospheric Thermodynamics. 2nd ed. D. Reidel, 259 pp.

Kuo, H. L., 1971: Axisymmetric flows in the boundary layer of a maintained vortex. J. Atmos. Sci., 28, 20-41.

LeeJoice, R. N., 2000: Hurricane inner-core structure as revealed by GPS dropwindsondes. M.S. thesis, Department of Atmospheric Science, Colorado State University, $56 \mathrm{pp}$.

Lilly, D. K., 1969: Tornado dynamics. NCAR Manuscript 69-117, $58 \mathrm{pp}$.

Long, R. R., 1953: Some aspects of the flow of stratified fluids. I. A theoretical investigation. Tellus, 5, 42-58.

Marks, F. D., P. G. Black, M. T. Montgomery, and R. W. Burpee, 2008: Structure of the eye and eyewall of Hurricane Hugo (1989). Mon. Wea. Rev., 136, 1237-1259.

Ooyama, K. V., 1982: Conceptual evolution of the theory and modeling of the tropical cyclone. J. Meteor. Soc. Japan, 60, 369-379.

Persing, J., and M. T. Montgomery, 2003: Hurricane superintensity. J. Atmos. Sci., 60, 2349-2371.

$\longrightarrow$, and —, 2005: Is environmental CAPE important in the determination of maximum possible hurricane intensity? J. Atmos. Sci., 62, 542-550.

Rott, N., and W. S. Lewellen, 1966: Boundary layers and their interactions in rotating flows. Progress in Aeronautical Sciences, 
A. Ferri, D. Küchmann, and L. H. G. Sterne, Eds., Pergamon Press, 111-144.

Rotunno, R., and K. A. Emanuel, 1987: An air-sea interaction theory for tropical cyclones. Part II: Evolutionary study using a nonhydrostatic axisymmetric numerical model. J. Atmos. Sci., 44, 542-561.

Schlichting, H., 1979: Boundary-Layer Theory. 7th ed. McGrawHill, 817 pp.
Smith, R. K., M. T. Montgomery, and S. Vogl, 2008: A critique of Emanuel's hurricane model and potential intensity theory. Quart. J. Roy. Meteor. Soc., 134, 551-561.

- ——, and N. V. Sang, 2009: Tropical cyclone spinup revisited. Quart. J. Roy. Meteor. Soc., 135, 1321-1335.

Yang, B., Y. Wang, and B. Wang, 2007: The effect of internally generated inner-core asymmetries on tropical cyclone potential intensity. J. Atmos. Sci., 64, 1165-1188. 